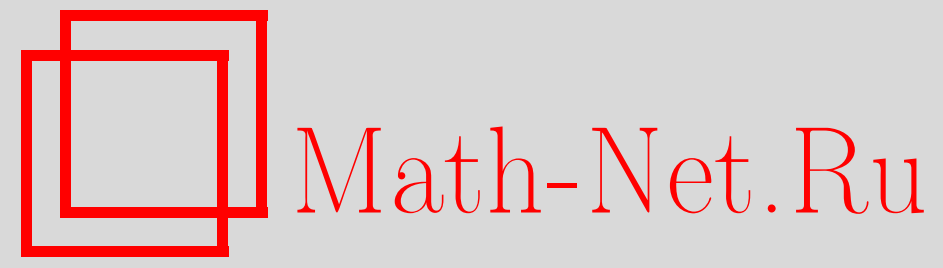

S. O'Rourke, D. Renfrew, A. Soshnikov, Fluctuations of matrix entries of regular functions of sample covariance random matrices, Теория вероятн. и ее примен., 2013, том 58, выпуск 4, 752-780

DOI: https://doi.org/10.4213/tvp4539

Использование Общероссийского математического портала Math-Net.Ru подразумевает, что вы прочитали и согласны с пользовательским соглашением http://www . mathnet.ru/rus/agreement

Параметры загрузки:

IP : 18.207 .199 .55

26 апреля 2023 г., $17: 38: 21$

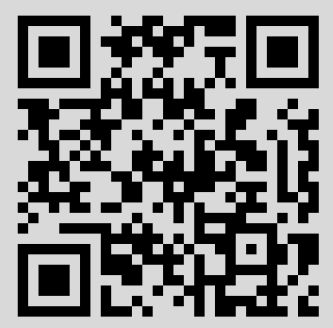




\title{
FLUCTUATIONS OF MATRIX ENTRIES OF REGULAR FUNCTIONS OF SAMPLE COVARIANCE RANDOM MATRICES ${ }^{1)}$
}

\begin{abstract}
Мы расширили результаты [19], [18], [17] о флуктуации матричных элементов регулярных функций матриц Вигнера на случай выборочных ковариационных матриц.

Ключевые слова и фразы: выборочные ковариационные матрицы, центральная предельная теорема, флуктуация матричных элементов.
\end{abstract}

1. Introduction and main results. Recently, there have been a number of results concerning matrix entries of functions of random matrices. That is, for a $N \times N$ random real symmetric (Hermitian) matrix, $M_{N}$, we consider the entries of the matrix $f\left(M_{N}\right)$ where $f: \mathbf{R} \rightarrow \mathbf{R}$ is a regular test function. We recall that for $M$ real symmetric (Hermitian), the matrix $f(M)$ is given by the functional calculus. Thus, given the spectral representation $M=V^{*} D V$ with $V$ an orthogonal (unitary) matrix and $D$ a diagonal matrix we have $f(M):=V^{*} f(D) V$, where $f(D)$ is again a diagonal matrix with $f(D)_{i i}=f\left(D_{i i}\right)$ on the diagonal. The $(i j)$-th entry of $f(M)$ is denoted by $f(M)_{i j}$.

In [14], Lytova and Pastur consider the case where $M_{N}$ is drawn from the Gaussian Orthogonal Ensemble (GOE) or Gaussian Unitary Ensemble (GUE). We recall that a GOE matrix is defined as $M_{N}=\left(Y_{N}+Y_{N}^{t}\right) / \sqrt{N}$, where the entries of $Y_{N}$ are i.i.d. $N\left(0, \sigma^{2} / 2\right)$ real random variables (see, e.g., [1]). In a similar way, a GUE matrix is defined as $M_{N}=\left(Y_{N}+Y_{N}^{*}\right) / \sqrt{N}$, where the entries of $X_{N}$ are i.i.d. $N\left(0, \sigma^{2} / 2\right)$ complex random variables. It was shown in [14] that

$$
\sqrt{N}\left(f\left(M_{N}\right)_{i j}-\mathbf{E}\left[f\left(M_{N}\right)_{i j}\right]\right) \longrightarrow N\left(0, \frac{1+\delta_{i j}}{\beta} \omega^{2}(f)\right),
$$

*Department of Mathematics, Rutgers University, 110 Frelinghuysen Rd. Piscataway, NJ 08854-8019, e-mail: sdo21@math.rutgers.edu

** Department of Mathematics, University of California, Davis, One Shields Avenue, Davis, CA 95616-8633, e-mail: drenfrew@math.ucdavis.edu; soshniko@math.ucdavis.edu

1) This work was supported by the NSF grants VIGRE DMS-0636297, DMS-1007558, and DMS-0905988 
in the limit when the size of the matrix goes to infinity, where $\omega^{2}(f)=$ $\mathbf{V}(f(\psi)), \psi$ is a random variable distributed according to the Wigner semicircle law, and $\beta=1$ for the GOE and $\beta=2$ for the GUE. We recall that the Wigner semicircle distribution is supported on the interval $[-2 \sigma, 2 \sigma]$ and its density with respect to the Lebesgue measure is given by

$$
\frac{d \mu_{s c}}{d x}(x)=\frac{1}{2 \pi \sigma^{2}} \sqrt{4 \sigma^{2}-x^{2}} \mathbf{1}_{[-2 \sigma, 2 \sigma]}(x) .
$$

In the case where $W_{N}=A_{N} / \sqrt{N}$, and $A_{N}$ is a symmetric (Hermitian) Wigner matrix ([1], [5]) with i.i.d. (not necessarily Gaussian) entries up from the diagonal, Pizzo, Renfrew, and Soshnikov studied in [19] the fluctuations of both the diagonal and off-diagonal entries under the condition that the off-diagonal entries of $A_{N}$ are centered and have finite fourth moment, and the diagonal entries of $A_{N}$ are centered and have finite second moment. The variance of the off-diagonal entries, as before, is equal to $\sigma^{2}$. The test function $f$ has been assumed to be four times continuously differentiable. In particular, it is shown in [19] that

$$
\sqrt{N}\left(f\left(W_{N}\right)_{i j}-\mathbf{E}\left[f\left(W_{N}\right)_{i j}\right]\right)
$$

converges in distribution to the sum of two independent random variables: the first (up to scaling) is given by $\left(A_{N}\right)_{i j}$ and the second is a Gaussian random variable with mean zero and variance explicitly given in terms of the function $f$. In addition, it was proven in [19] that the joint distribution of any finite number of normalized matrix entries converges to the product of one-dimensional limiting distributions. If the marginal distribution of the entries of $W_{N}$ is Gaussian (so $W_{N}$ belongs to the GOE (GUE) ensemble), one recovers (1.1).

Such results might be considered as an analogue of the Borel theorem for the matrix entries of random matrices from the classical compact groups (see, e.g., [8], [11], and [12]). In addition, the results about the fluctuation of the resolvent quadratic form are related to the limiting distribution of the outliers in the spectrum of finite rank deformations of Wigner matrices (see, e.g., [20] and references therein).

Almost simultaneously with [19] and using a different set of ideas, Pastur and Lytova [18] gave another proof of the limiting distribution of the normalized diagonal entries

$$
\sqrt{N}\left(f\left(W_{N}\right)_{i i}-\mathbf{E}\left[f\left(W_{N}\right)_{i i}\right]\right), \quad 1 \leqslant i \leqslant N,
$$

when $W_{N}=A_{N} / \sqrt{N}$, and $A_{N}$ is a real symmetric Wigner matrix with i.i.d. centered entries up from the diagonal provided the cumulant generating function $\ln \left(\mathbf{E} \exp \left(z A_{12}\right)\right)$ is entire (so, in particular, all moments of the 
marginal distribution are finite) and the test function $f$ satisfies

$$
\int_{\mathbf{R}}(1+2|k|)^{3}|\hat{f}(k)| d k<\infty
$$

where $\hat{f}$ is the Fourier transform

$$
\hat{f}(k)=\frac{1}{\sqrt{2 \pi}} \int_{\mathbf{R}} e^{-i k x} f(x) d x .
$$

The results of [19] and [18] are extended in [17] to the case of a Wigner matrix with non-i.i.d. entries, where it was assumed that the off-diagonal entries have uniformly bounded fourth moments, diagonal entries have uniformly bounded second moments, and certain Lindeberg type conditions for the fourth moments of the off-diagonal entries and the second moments of the diagonal entries are satisfied. The test function $f(x)$ is assumed to satisfy

$$
\int_{\mathbf{R}}(1+2|k|)^{2 s}|\hat{f}(k)|^{2} d k<\infty
$$

for some $s>3$.

In this paper, we study the fluctuations of matrix entries of a sample covariance random matrix. Namely, we consider the case where

$$
M_{N}=\frac{1}{N} A_{N} A_{N}^{*}
$$

and $A_{N}$ is an $N \times n$ rectangular matrix with independent entries. We begin with some definitions.

D e f i n i t i o n 1.1. Let $A_{N}=\left(\left(A_{N}\right)_{i j}\right)_{1 \leqslant i \leqslant N ; 1 \leqslant j \leqslant n}$ be an $N \times n$ matrix with complex entries. We say the matrix $A_{N}$ satisfies condition C1 if

(i) $\left\{\operatorname{Re}\left(A_{N}\right)_{i j}, \operatorname{Im}\left(A_{N}\right)_{i j}: 1 \leqslant i \leqslant N ; 1 \leqslant j \leqslant n\right\}$ is a collection of independent random variables,

(ii) each entry $\left(A_{N}\right)_{i j}$ has mean 0 and variance $\sigma^{2}$,

(iii) each entry satisfies $\mathbf{E}\left(A_{N}\right)_{i, j}^{2}=0$,

(iv) $\sup _{N, i, j} \mathbf{E}\left|\left(A_{N}\right)_{i j}\right|^{4}=m_{4}<\infty$,

(v) the entries satisfy the Lindeberg condition for the fourth moments, that is, for all $\varepsilon>0$,

$$
\frac{1}{N^{2}} \sum_{i, j} \mathbf{E}\left|\left(A_{N}\right)_{i j}\right|^{4} \mathbf{1}_{\left\{\left|\left(A_{N}\right)_{i j}\right|>\varepsilon \sqrt{N}\right\}} \longrightarrow 0
$$

as $N \rightarrow \infty$.

D e f i n i t i o n 1.2. Let $A_{N}=\left(\left(A_{N}\right)_{i j}\right)_{1 \leqslant i \leqslant N ; 1 \leqslant j \leqslant n}$ be an $N \times n$ matrix with real entries. We say the matrix $A_{N}$ satisfies condition C2 if $\left\{\left(A_{N}\right)_{i j}: 1 \leqslant i \leqslant N ; 1 \leqslant j \leqslant n\right\}$ is a collection of independent real random variables and conditions (ii), (iv), and (v) hold from Definition 1.1. 
We define $X_{N}:=A_{N} / \sqrt{N}$ and $M_{N}:=X_{N} X_{N}^{*}$. Throughout this paper, we assume that $c_{N}:=n / N \rightarrow c \in(0, \infty)$ as $N \rightarrow \infty$.

$\mathrm{D}$ e f i n i t i o n 1.3. Let $B$ be an $N \times N$ self-adjoint matrix with eigenvalues $\lambda_{1}, \ldots, \lambda_{N}$. The empirical spectral density of $B$ is given by

$$
\mu_{B}:=\frac{1}{N} \sum_{i=1}^{N} \delta_{\lambda_{i}}
$$

The limiting empirical spectral density of $M_{N}$ is known as the Marchenko-Pastur law (see [3], [16]).

Theorem 1.1 (Marchenko-Pastur). Suppose that for each $N$, the entries of $A_{N}$ are independent complex (real) random variables with mean 0 and variance $\sigma^{2}$. Assume $n / N \rightarrow c \in(0, \infty)$ and for any $\varepsilon>0$

$$
\frac{1}{N^{2}} \sum_{i, j} \mathbf{E}\left|\left(A_{N}\right)_{i j}\right|^{2} \mathbf{1}_{\left\{\left|\left(A_{N}\right)_{i j}\right|>\varepsilon \sqrt{N}\right\}} \longrightarrow 0
$$

as $N \rightarrow \infty$. Then with probability one, the empirical density $\mu_{M_{N}}$ tends to the Marchenko-Pastur distribution, $\mu_{\sigma, c}$, with ratio index $c$ and scale index $\sigma^{2}$, where

$$
\frac{d \mu_{\sigma, c}}{d x}(x)=\left\{\begin{array}{lr}
\left(2 \pi x \sigma^{2}\right)^{-1} \sqrt{\left(u_{+}-x\right)\left(x-u_{-}\right)}, & u_{-} \leqslant x \leqslant u_{+} \\
0, & \text { otherwise }
\end{array}\right.
$$

with a point mass at 0 with weight $(1-c)$ when $c<1$, and where

$$
u_{+}:=\sigma^{2}(1+\sqrt{c})^{2}, \quad u_{-}:=\sigma^{2}(1-\sqrt{c})^{2} .
$$

$\mathrm{R}$ e $\mathrm{m}$ a $\mathrm{r} \mathrm{k}$ 1.1. We note that the Lindeberg condition (1.6) is implied by the Lindeberg condition for the fourth moments (1.5).

Given a probability measure $\mu$ on the real line, its Stieltjes transform is given by

$$
\int_{\mathbf{R}} \frac{d \mu(x)}{z-x}, \quad z \in \mathbb{C} \backslash \operatorname{supp}(\mu)
$$

For $\operatorname{Im} z \neq 0$, we have the following bound for the Stieltjes transform of any probability measure on $\mathbf{R}$

$$
\left|\int_{\mathbf{R}} \frac{d \mu(x)}{z-x}\right| \leqslant \frac{1}{|\operatorname{Im}(z)|}
$$

The Stieltjes transform of $\mu_{\sigma, c}$ is denoted by $g_{\sigma, c}$ and is characterized as the solution of

$$
z \sigma^{2} g_{\sigma, c}(z)+\left(\sigma^{2}(c-1)-z\right) g_{\sigma, c}(z)+1=0
$$

that decays to zero as $z \rightarrow \infty$. 
The Stieltjes transform of the expectation of the empirical spectral distribution of $M_{N}$ is given by

$$
g_{N}(z)=\mathbf{E} \int_{\mathbf{R}} \frac{d \mu_{M_{N}}(x)}{z-x}=\mathbf{E}\left[\operatorname{tr}_{N}\left(R_{N}(z)\right)\right],
$$

where $\operatorname{tr}_{N}:=\operatorname{Tr} / N$ is the normalized trace and $R_{N}(z):=\left(z I_{N}-M_{N}\right)^{-1}$ is the resolvent of $M_{N}$. If it does not lead to ambiguity, we will use the shorthand notation $R_{i j}(z)$ for $\left(R_{N}(z)\right)_{i j}, 1 \leqslant i, j \leqslant N$.

For $s \geqslant 0$, we consider the space $\mathscr{H}_{s}$ consisting of the functions $\phi: \mathbf{R} \rightarrow$ $\mathbf{R}$ that satisfy

$$
\|\phi\|_{s}^{2}:=\int_{\mathbf{R}}(1+2|k|)^{2 s}|\widehat{\phi}(k)|^{2} d k<\infty .
$$

We recall that $C^{k}(X)$ denotes the space of $k$ times continuously differentiable functions on $X \subset \mathbf{R}$ and define the $C^{k}(X)$ norm

$$
\|\phi\|_{C^{k}(X)}:=\max \left(\left|\frac{d^{l} f(x)}{d x^{l}}\right|, x \in X, 0 \leqslant l \leqslant k\right) .
$$

We now present our main results.

Theorem 1.2. Let $A_{N}$ be a $N \times n$ random matrix with real entries that satisfies condition $\mathbf{C 2}$. Let $m$ be a fixed positive integer and assume that for $1 \leqslant i \leqslant m$

$$
m_{4}(i):=\lim _{N \rightarrow \infty} \frac{1}{n} \sum_{j=1}^{n} \mathbf{E}\left|A_{i j}\right|^{4}
$$

exists and for all $\varepsilon>0$

$$
\frac{1}{N} \sum_{j=1}^{n} \mathbf{E}\left|\left(A_{N}\right)_{i j}\right|^{4} \mathbf{1}_{\left\{\left|\left(A_{N}\right)_{i j}\right|>\varepsilon N^{1 / 4}\right\}} \longrightarrow 0, \quad 1 \leqslant i \leqslant m,
$$

as $N \rightarrow \infty$. Assume $c_{N} \rightarrow c \in(0, \infty)$ as $N \rightarrow \infty$ and let $f \in \mathscr{H}_{s}$ for some $s>3$. Then we have the following:

(i) The normalized matrix entries

$$
\left\{\sqrt{N}\left(f\left(M_{N}\right)_{i j}-\mathbf{E}\left[f\left(M_{N}\right)_{i j}\right]\right): 1 \leqslant i \leqslant j \leqslant m\right\}
$$

are independent in the limit $N \rightarrow \infty$.

(ii) For $1 \leqslant i<j \leqslant m$,

$$
\sqrt{N}\left(f\left(M_{N}\right)_{i j}-\mathbf{E}\left[f\left(M_{N}\right)_{i j}\right]\right) \longrightarrow N\left(0, \omega^{2}(f)\right)
$$

in distribution as $N \rightarrow \infty$, where

$$
\omega^{2}(f)=\mathbf{V}\left(f\left(\eta_{c}\right)\right)
$$


and $\eta_{c}$ is a Marchenko-Pastur distributed random variable with ratio index $c$ and scale index $\sigma^{2}$.

(iii) For $1 \leqslant i \leqslant m$,

$$
\left.\sqrt{N}\left(f\left(M_{N}\right)_{i i}-\mathbf{E}\left[f\left(M_{N}\right)_{i i}\right]\right) \rightarrow N\left(0,2 \omega^{2}(f)+\frac{\kappa_{4}(i)}{\sigma^{4}} \rho^{2}(f)\right)\right)
$$

in distribution as $N \rightarrow \infty$, where

$$
\rho(f)=\mathbf{E}\left[f\left(\eta_{c}\right) \frac{\eta_{c}-c \sigma^{2}}{\sqrt{c} \sigma^{2}}\right]
$$

and

$$
\kappa_{4}(i):=m_{4}(i)-3 \sigma^{4} .
$$

Theorem 1.3. Let $A_{N}$ be a $N \times n$ random matrix with complex entries that satisfies condition $\mathbf{C 1}$. Let $m$ be a fixed positive integer and assume that for $1 \leqslant i \leqslant m$

$$
m_{4}(i):=\lim _{N \rightarrow \infty} \frac{1}{n} \sum_{j=1}^{n} \mathbf{E}\left|A_{i j}\right|^{4}
$$

exists and for all $\varepsilon>0$ (1.11) holds as $N \rightarrow \infty$. Assume $c_{N} \rightarrow c \in(0, \infty)$ as $N \rightarrow \infty$ and let $f \in \mathscr{H}_{s}$ for some $s>3$. Then we have the following:

(i) The normalized matrix entries

$$
\left\{\sqrt{N}\left(f\left(M_{N}\right)_{i j}-\mathbf{E}\left[f\left(M_{N}\right)_{i j}\right]\right): 1 \leqslant i \leqslant j \leqslant m\right\}
$$

are independent in the limit $N \rightarrow \infty$.

(ii) For $1 \leqslant i<j \leqslant m$,

$$
\sqrt{N}\left(f\left(M_{N}\right)_{i j}-\mathbf{E}\left[f\left(M_{N}\right)_{i j}\right]\right) \longrightarrow N\left(0, \omega^{2}(f)\right)
$$

in distribution as $N \rightarrow \infty$, where $N\left(0, \omega^{2}(f)\right)$ stands for the complex Gaussian random variable with i.i.d. real and imaginary parts with variance $\omega^{2}(f) / 2$ and $\omega(f)$ is defined in (1.12).

(iii) For $1 \leqslant i \leqslant m$,

$$
\sqrt{N}\left(f\left(M_{N}\right)_{i i}-\mathbf{E}\left[f\left(M_{N}\right)_{i i}\right]\right) \rightarrow N\left(0, \omega^{2}(f)+\frac{\kappa_{4}(i)}{\sigma^{4}} \rho^{2}(f)\right)
$$

in distribution as $N \rightarrow \infty$, where $\omega(f)$ is defined in (1.12), $\rho(f)$ is defined in (1.13), and $\kappa_{4}(i):=m_{4}(i)-2 \sigma^{4}$.

$\mathrm{R}$ e $\mathrm{m}$ a $\mathrm{rk}$ 1.2. The limiting distribution of an entry in the sample covariance case is Gaussian and differs from the Wigner case ([18], [19]), where the limiting distribution is given by a linear combination of an independent Gaussian random variable and the corresponding entry of the 
Wigner matrix. However, in the square case $(c=1)$ the limiting distribution of $\sqrt{N}\left(f\left(M_{N}\right)_{i j}-\mathbf{E}\left[f\left(M_{N}\right)_{i j}\right]\right)$ coincides with the limiting distribution of $\sqrt{N}\left(g\left(W_{N}\right)_{i j}-\mathbf{E}\left[g\left(W_{N}\right)_{i j}\right]\right)$, where $g(x)=f\left(x^{2}\right)$ and $W_{N}$ is a Wigner random matrix. This is not surprising since $M_{N}$ is the $N \times N$ upper-left corner submatrix of the $(N+n) \times(N+n)$ matrix $Z_{N, n}^{2}$, where the $N \times N$ upper-left and $n \times n$ lower-right corner submatrices of $Z_{N, n}$ are both zero, the $N \times n$ upper-right corner submatrix of $Z_{N, n}$ is given by $X_{N}$, and the $n \times N$ lower-left corner submatrix of $Z_{N, n}$ is given by $X_{N}^{*}$. The limiting spectral distribution of $Z_{N, n}$ in the case $n / N \rightarrow c=1$ is given by the Wigner semicircle law and the technique of [19], [17] in the square case can be extended without any difficulties to $Z_{N, n}$.

$\mathrm{R} \mathrm{e} \mathrm{m}$ a r k 1.3. The functions 1 and $\left(x-c \sigma^{2}\right) /\left(\sqrt{c} \sigma^{2}\right)$ are the first two orthonormal polynomials with respect to $\mu_{\sigma, c}(d x)$. Therefore, by the Bessel inequality, the variance of the limiting Gaussian distribution for the diagonal entries is zero if and only if the test function is linear and the marginal distribution is Bernoulli. For the off-diagonal entries, it immediately follows from (1.12) that the variance is zero if and only if the test function is constant on the support of the Marchenko-Pastur law.

$\mathrm{R}$ e $\mathrm{m}$ a r k 1.4. It follows from Proposition 4.1 and Lemma 2.2 that if $f \in C^{7}(\mathbf{R})$ for the diagonal entries $i=j\left(f \in C^{6}(\mathbf{R})\right.$ in the off-diagonal case $i \neq j)$, one can replace $\mathbf{E}\left[f\left(M_{N}\right)_{i j}\right]$ in Theorems 1.2 and 1.3 by $\delta_{i j} \times$ $\int f(x) d \mu_{\sigma, c_{N}}(x)$. Moreover, as shown in Proposition 4.1, if $\operatorname{supp}(f) \cap R_{+}$is compact, where $R^{+}=[0, \infty)$ and $f$ has seven continuous derivatives, then $\mathbf{E}\left[f\left(M_{N}\right)_{i i}\right]=\int f(x) d \mu_{\sigma, c_{N}}(x)+O(1 / N)$. If $f$ has six bounded continuous derivatives on $R_{+}$, then $\mathbf{E}\left[f\left(M_{N}\right)_{i j}\right]=O(1 / N), i \neq j$.

We divide the proof of Theorems 1.2 and 1.3 into several sections. In Section 2, we apply a standard truncation lemma to the matrix entries of $A_{N}$. Section 3 is devoted to computing the expectation and variance of the entries of the resolvent, $R_{N}(z)$, and Section 4 extends these results to more general functions. In Section 5, we prove a central limit theorem for entries of $f\left(M_{N}\right)$ where $f(x)$ is a finite linear combination of the functions $(z-x)^{-1}, z \in \mathbb{C} \backslash \mathbf{R}$. Finally, we extend this result to more general test functions $f \in \mathscr{H}_{s}$ by an approximation argument.

2. Truncation and extremal eigenvalues. We note that by (1.5), we can choose a sequence $\varepsilon_{N} \rightarrow 0$ such that

$$
\frac{1}{\varepsilon_{N}^{4} N^{2}} \sum_{i, j} \mathbf{E}\left|\left(A_{N}\right)_{i j}\right|^{4} \mathbf{1}_{\left\{\left|\left(A_{N}\right)_{i j}\right|>\varepsilon_{N} \sqrt{N}\right\}} \longrightarrow 0
$$

as $N \rightarrow \infty$.

Lemma 2.1. Assume that $A_{N}$ is an $N \times n$ matrix that satisfies condition $\mathbf{C 1}$ in the complex case (condition $\mathbf{C 2}$ in the real case). Then there 
exists a random $N \times n$ matrix $\widetilde{A}_{N}$ with independent entries and a sequence $\varepsilon_{N}$ which tends to zero as $N$ tends to infinity such that

(i) the entries $\left(\widetilde{A}_{N}\right)_{i j}$ have mean zero and variance $\sigma^{2}$,

(ii) $\sup _{i, j}\left|\left(\widetilde{A}_{N}\right)_{i j}\right| \leqslant \varepsilon_{N} \sqrt{N}$,

(iii) $\sup _{N, i, j} \mathbf{E}\left|\left(\widetilde{A}_{N}\right)_{i j}\right|^{4}<\infty$,

(iv) $\mathbf{P}\left(A_{N} \neq \widetilde{A}_{N}\right) \longrightarrow 0$ as $N \rightarrow \infty$.

$\mathrm{Pr}$ o of. We present the proof in the case where the entries of $A_{N}$ are real. The complex case follows a similar argument. We begin by selecting a sequence $\varepsilon_{N} \rightarrow 0$ such that $(2.1)$ holds. Then let $\left(\widehat{A}_{N}\right)_{i j}=$ $\left(A_{N}\right)_{i j} \mathbf{1}_{\left\{\left|\left(A_{N}\right)_{i j}\right| \leqslant \varepsilon_{N} \sqrt{N}\right\}}$. Define $m_{N i j}=\mathbf{E}\left(\widehat{A}_{N}\right)_{i j}, v_{N i j}^{2}=\sigma^{2}-\mathbf{E}\left(\widehat{A}_{N}\right)_{i j}^{2}$. Then we have that

$$
\begin{aligned}
\left|m_{N i j}\right| & \leqslant \mathbf{E}\left|\left(A_{N}\right)_{i j}\right| \mathbf{1}_{\left\{\left|\left(A_{N}\right)_{i j}\right|>\varepsilon_{N} \sqrt{N}\right\}} \\
& \leqslant \frac{\mathbf{E}\left|\left(A_{N}\right)_{i j}\right|{ }^{4} \mathbf{1}_{\left\{\left|\left(A_{N}\right)_{i j}\right|>\varepsilon_{N} \sqrt{N}\right\}}}{\varepsilon_{N}^{3} N^{3 / 2}}=O\left(\frac{1}{\varepsilon_{N}^{3} N^{3 / 2}}\right)
\end{aligned}
$$

and similarly

$$
\begin{gathered}
v_{N i j}^{2} \leqslant \mathbf{E}\left|\left(A_{N}\right)_{i j}\right|^{2} \mathbf{1}_{\left\{\left|\left(A_{N}\right)_{i j}\right|>\varepsilon_{N} \sqrt{N}\right\}} \leqslant O\left(\frac{1}{\varepsilon_{N}^{2} N}\right), \\
\left|m_{N i j}\right| \leqslant \frac{v_{N i j}^{2}}{\varepsilon_{N} N^{1 / 2}} .
\end{gathered}
$$

We now define $\left(\widetilde{A}_{N}\right)_{i j}$ to be a mixture of

(1) $\left(\widehat{A}_{N}\right)_{i j}$ with probability $1-\left|m_{N i j}\right| /\left(\varepsilon_{N} \sqrt{N}\right)-v_{N i j}^{2} /\left(\varepsilon_{N}^{2} N\right)$; and

(2) a Bernoulli random variable $\xi_{N i j}$ with probability $\left|m_{N i j}\right| /\left(\varepsilon_{N} \sqrt{N}\right)+$ $v_{N i j}^{2} /\left(\varepsilon_{N}^{2} N\right)$, where we denote the mean and the second moment of $\xi_{N i j}$ by $\mu_{N i j}$ and $\tau_{N i j}^{2}$.

We can choose $\xi_{N i j}$ such that

(i) $\mathbf{E}\left(\widetilde{A}_{N}\right)_{i j}=0$,

(ii) $\mathbf{V}\left(\widetilde{A}_{N}\right)_{i j}=\sigma^{2}$,

(iii) $\left(\widetilde{A}_{N}\right)_{i j} \leqslant C \varepsilon_{N} \sqrt{N}$ for some absolute constant $C$.

We now verify that such a construction is possible. Essentially, we have to show that one can choose $\xi_{N i j}$ in such a way that (i) and (ii) are satisfied and

$$
\left|\mu_{N i j}\right| \leqslant C_{1} \varepsilon_{N} \sqrt{N} \text { and } \tau_{N i j}^{2} \leqslant C_{2} \varepsilon_{N}^{2} N
$$

for some absolute constants $C_{1}, C_{2}>0$. Indeed, if this is the case, we can construct $\xi_{N i j}=\mu_{N i j}+\psi_{N i j}$, where $\psi_{N i j}$ is a symmetric Bernoulli random variable satisfying $\left|\psi_{N i j}\right| \leqslant C \varepsilon_{N} \sqrt{N}$, where $C$ is an absolute constant that depends on $C_{1}$ and $C_{2}$. This would immediately follow from (2.5). To verify (2.5), we note that

$$
0=m_{N i j}\left(1-\frac{\left|m_{N i j}\right|}{\varepsilon_{N} \sqrt{N}}-\frac{v_{N i j}^{2}}{\varepsilon_{N}^{2} N}\right)+\mu_{N i j}\left(\frac{\left|m_{N i j}\right|}{\varepsilon_{N} \sqrt{N}}+\frac{v_{N i j}^{2}}{\varepsilon_{N}^{2} N}\right),
$$




$$
\sigma^{2}=\left(\sigma^{2}-v_{N i j}^{2}\right)\left(1-\frac{\left|m_{N i j}\right|}{\varepsilon_{N} \sqrt{N}}-\frac{v_{N i j}^{2}}{\varepsilon_{N}^{2} N}\right)+\tau_{N i j}^{2}\left(\frac{\left|m_{N i j}\right|}{\varepsilon_{N} \sqrt{N}}+\frac{v_{N i j}^{2}}{\varepsilon_{N}^{2} N}\right) .
$$

Solving for $\mu_{N i j}$ in the first equation and $\tau_{N i j}$ in the second and applying (2.2)-(2.4) yields the required bounds (2.5), verifying the claim.

We note that without loss of generality we may assume $C=1$ by our choice of the sequence $\varepsilon_{N}$.

Next by (2.2) and (2.3), we have that

$$
\mathbf{E}\left|\left(\widetilde{A}_{N}\right)_{i j}\right|^{4} \leqslant \mathbf{E}\left|\left(A_{N}\right)_{i j}\right|^{4}+\left(\frac{\left|m_{N i j}\right|}{\varepsilon_{N} \sqrt{N}}+\frac{v_{N i j}^{2}}{\varepsilon_{N}^{2} N}\right)\left(\varepsilon_{N} \sqrt{N}\right)^{4} \leqslant m_{4}+O(1) .
$$

To complete the proof of Lemma 2.1, we apply (2.2), (2.3), and (2.1) to obtain

$$
\begin{aligned}
\mathbf{P}\left(\widetilde{A}_{N} \neq A_{N}\right) & \leqslant \sum_{i, j}\left(\frac{\left|m_{N i j}\right|}{\varepsilon_{N} \sqrt{N}}+\frac{v_{N i j}^{2}}{\varepsilon_{N}^{2} N}+\mathbf{P}\left(\left|\left(A_{N}\right)_{i j}\right|>\varepsilon_{N} \sqrt{N}\right)\right) \\
& \leqslant \frac{3}{\varepsilon_{N}^{4} N^{2}} \sum_{i, j} \mathbf{E}\left|\left(A_{N}\right)_{i j}\right|^{4} \mathbf{1}_{\left\{\left|\left(A_{N}\right)_{i j}\right|>\varepsilon_{N} \sqrt{N}\right\}} \longrightarrow 0
\end{aligned}
$$

as $N \rightarrow \infty$.

We can now apply Lemma 2.1 to obtain a result on the norm of the matrix $A_{N} A_{N}^{*} / N$. This result follows from [3, Theorem 5.9]. We present it here for completeness.

Lemma 2.2. Under the assumptions of Lemma 2.1, we have that $\left\|A_{N} A_{N}^{*} / N\right\| \longrightarrow \sigma^{2}(1+\sqrt{c})^{2}$ in probability as $N \rightarrow \infty$.

P r o of. Since Theorem 5.9 from [3] does not apply directly to $A_{N} A_{N}^{*} / N$, we simply note that by Lemma 2.1 , it is enough to show $\left\|\widetilde{A}_{N} \widetilde{A}_{N}^{*} / N\right\| \longrightarrow \sigma^{2}(1+\sqrt{c})^{2}$ in probability. Theorem 5.9 from [3] now applies to the matrix $\widetilde{A}_{N} \widetilde{A}_{N}^{*} / N$ to obtain

$$
\mathbf{P}\left(\left\|\frac{1}{N} \widetilde{A}_{N} \widetilde{A}_{N}^{*}\right\|>\sigma^{2}(1+\sqrt{c})^{2}+x\right) \longrightarrow 0
$$

as $N \rightarrow \infty$ for all $x>0$. The proof is then complete by noting that Theorem 1.1 implies that, with probability 1 ,

$$
\limsup _{N \rightarrow \infty}\left\|\frac{1}{N} \widetilde{A}_{N} \widetilde{A}_{N}^{*}\right\| \geqslant \sigma^{2}(1+\sqrt{c})^{2} .
$$

We also note that by $(1.11)$, we can choose a sequence $\varepsilon_{N} \rightarrow 0$ such that

$$
\frac{1}{\varepsilon_{N}^{4} N} \sum_{j=1}^{n} \mathbf{E}\left|\left(A_{N}\right)_{i j}\right|^{4} \mathbf{1}_{\left\{\left|\left(A_{N}\right)_{i j}\right|>\varepsilon_{N} N^{1 / 4}\right\}} \longrightarrow 0
$$

as $N \rightarrow \infty$ for $1 \leqslant i \leqslant m$. 
Lemma 2.3. Let $A_{N}$ be a $N \times n$ complex (real) matrix that satisfies condition $\mathbf{C 1}$ (C2) and (2.6) for $1 \leqslant i \leqslant m$, where $m$ is a fixed positive integer. Then there exists a random $N \times n$ matrix $\widetilde{A}_{N}$ with independent entries and a sequence $\varepsilon_{N}$ which tends to zero as $N$ tends to infinity such that

(i) $\left(\widetilde{A}_{N}\right)_{i j}=\left(A_{N}\right)_{i j}$ for $m<i \leqslant N$ and $1 \leqslant j \leqslant n$,

(ii) the entries $\left(\widetilde{A}_{N}\right)_{i j}$ have mean zero and variance $\sigma^{2}$,

(iii) $\sup _{j, 1 \leqslant i \leqslant m}\left|\left(\widetilde{A}_{N}\right)_{i j}\right| \leqslant \varepsilon_{N} N^{1 / 4}$,

(iv) $\sup _{N, i, j} \mathbf{E}\left|\left(\widetilde{A}_{N}\right)_{i j}\right|^{4}<\infty$,

(v) $\mathbf{P}\left(A_{N} \neq \widetilde{A}_{N}\right) \longrightarrow 0$ as $N \rightarrow \infty$,

(vi) $\frac{1}{n} \sum_{j}\left(\mathbf{E}\left|A_{i j}\right|^{4}-\mathbf{E}\left|\widetilde{A}_{i j}\right|^{4}\right) \rightarrow 0,1 \leqslant i \leqslant m$.

The proof of Lemma 2.3 is very similar to the proof of Lemma 2.1 and the details are left to the reader.

By Lemmas 2.1 and 2.3, we will assume that all the entries of $A_{N}$ are bounded by $\varepsilon_{N} \sqrt{N}$ and that the entries satisfy conditions (ii)-(iv) of Lemma 2.3 for the remainder of the paper. Indeed, since the truncated matrix coincides with the original with probability going to 1 , it is enough for us to prove Theorems 1.2 and 1.3 for the truncated matrix.

We will also need the following lemma for controlling the expectation of the norm of $X_{N} X_{N}^{*}$.

Lemma 2.4. For any $k \geqslant 1$, there exists a constant $C>0$ (depending only on $\sigma, c$, and $k$ ) such that

$$
\mathbf{E}\left[\left\|X_{N} X_{N}^{*}\right\|^{k}\right] \leqslant C
$$

for $N$ sufficiently large.

$\mathrm{P}$ r o o f. For any $\varepsilon>0$,

$$
\begin{aligned}
\mathbf{E}\left[\left\|X_{N} X_{N}^{*}\right\|^{k}\right]= & \mathbf{E}\left[\left\|X_{N} X_{N}^{*}\right\|^{k} \mathbf{1}_{\left\{\left\|X_{N} X_{N}^{*}\right\| \leqslant \sigma^{2}(1+\sqrt{c})^{2}+\varepsilon\right\}}\right] \\
& +\mathbf{E}\left[\left\|X_{N} X_{N}^{*}\right\|^{k} \mathbf{1}_{\left\{\left\|X_{N} X_{N}^{*}\right\|>\sigma^{2}(1+\sqrt{c})^{2}+\varepsilon\right\}}\right] \\
\leqslant & {\left[\sigma^{2}(1+\sqrt{c})^{2}+\varepsilon\right]^{k} } \\
& +k \int_{\varepsilon}^{\infty} t^{k-1} \mathbf{P}\left(\left\|X_{N} X_{N}^{*}\right\|>t+\sigma^{2}(1+\sqrt{C})^{2}\right) d t .
\end{aligned}
$$

By [3, Theorem 5.9], we have

$$
\begin{aligned}
\int_{\varepsilon}^{\infty} & t^{k-1} \mathbf{P}\left(\left\|X_{N} X_{N}^{*}\right\|>t+\sigma^{2}(1+\sqrt{C})^{2}\right) d t \\
& \leqslant C^{\prime} N^{-k-2} \int_{\varepsilon}^{\infty} t^{k-1}\left(\sigma^{2}(1+\sqrt{C})^{2}+t-\varepsilon\right)^{-k-2} d t=O\left(N^{-k-2}\right)
\end{aligned}
$$

for some constant $C^{\prime}>0$. Thus,

$$
\mathbf{E}\left[\left\|X_{N} X_{N}^{*}\right\|^{k}\right] \leqslant\left[\sigma^{2}(1+\sqrt{c})^{2}+\varepsilon\right]^{k}+1
$$

for $N$ sufficiently large. Lemma 2.4 is proved. 


\section{Mathematical expectation and variance of resolvent entries.}

This section is devoted to the estimates of the mathematical expectation and the variance of the resolvent entries. Throughout the section, we will consider the real case. The proofs in the complex case are very similar. It follows from Lemmas 2.1 and 2.3 that for the purposes of the proof of Theorems 1.2 and 1.3 we can assume that $A_{N}$ satisfies properties (i)-(iii) in Lemma 2.1 and properties (ii)-(iv) in Lemma 2.3. Indeed, such a truncated matrix coincides with $A_{N}$ with probability going to 1 , and, therefore, if the results of Theorems 1.2 and 1.3 hold for the truncated matrix, they also hold for $A_{N}$.

We begin by recalling the basic resolvent identity

$$
\left(z I-A_{2}\right)^{-1}=\left(z I-A_{1}\right)^{-1}-\left(z I-A_{1}\right)^{-1}\left(A_{1}-A_{2}\right)\left(z I-A_{2}\right)^{-1},
$$

which holds for all $z \in \mathbb{C}$, where $\left(z I-A_{1}\right)$ and $\left(z I-A_{2}\right)$ are invertible.

We will also use the decoupling formula (see, for example, [13] and [15]): for any real-valued random variable, $\xi$, with $p+2$ finite moments and $\phi$ a complex-valued function with $p+1$ continuous and bounded derivatives the decoupling formula is given by:

$$
\mathbf{E}(\xi \varphi(\xi))=\sum_{a=0}^{p} \frac{\kappa_{a+1}}{a !} \mathbf{E}\left(\varphi^{(a)}(\xi)\right)+\varepsilon,
$$

where $\kappa_{a}$ are the cumulants of $\xi$ and $\varepsilon \leqslant C \sup _{t}\left|\varphi^{(p+1)}(t)\right| \mathbf{E}\left(|\xi|^{p+2}\right), C$ depends only on $p$. It follows from the proof of the decoupling formula in [15] that if $|\xi| \leqslant K$ with probability 1 , then the supremum in the upper bound for the error term can be taken over $t \in[-K, K]$.

Recall that we denote the entries of the resolvent $R_{N}(z)=\left(z I_{N}-M_{N}\right)^{-1}$ of $M_{N}$ by $R_{i j}(z), 1 \leqslant i, j \leqslant N$. Using (3.1), we can compute the derivatives of the resolvent with respect to any entry

$$
\frac{\partial R_{i j}}{\partial X_{k l}}=R_{i k}\left(X^{*} R\right)_{l j}+(R X)_{i l} R_{k j} .
$$

We now use (3.2) and (3.3) to compute the expectation and variance of the resolvent entries.

Proposition 3.1. Let $M_{N}=A_{N} A_{N}^{*} / N$ be a random real (complex) sample covariance matrix satisfying condition $\mathbf{C 2}(\mathbf{C 1})$ and $R_{N}(z)=\left(z I_{N}-\right.$ $\left.M_{N}\right)^{-1}$. Then

$$
\mathbf{E}\left[R_{i i}(z)\right]=g_{\sigma, c_{N}}(z)+O\left(\frac{P_{6}\left(|\operatorname{Im}(z)|^{-1}\right)}{N}\right)
$$

for $1 \leqslant i \leqslant N$, uniformly on bounded sets of $\mathbb{C} \backslash \mathbf{R}$

$$
\mathbf{E}\left[R_{i k}(z)\right]=O\left(\frac{P_{5}\left(|\operatorname{Im}(z)|^{-1}\right)}{N}\right)
$$


for $1 \leqslant i \neq k \leqslant N$, uniformly on $z \in \mathbb{C} \backslash \mathbf{R}$;

$$
\mathbb{V}\left[R_{i j}(z)\right]=O\left(\frac{P_{4}\left(|\operatorname{Im}(z)|^{-1}\right) \mathbf{E}\left[P_{10}\left(\left\|X_{N}\right\|\right)\left(\left\|R_{N}(z)\right\|^{2}+\left\|R_{N}(z)\right\|^{3 / 2}\right)\right]}{N}\right)
$$

for $1 \leqslant i, j \leqslant N$, uniformly on $z \in \mathbb{C} \backslash \mathbf{R}$.

Here and throughout the paper $P_{k}$ denotes a polynomial of degree $k$ with nonnegative coefficients.

In (3.6) we have included the norm of the resolvent in the error estimate. This will be useful in the proof of Proposition 4.6.

P r o o f. The following inequalities will be useful in our calculations:

$$
\left|R_{i k}(z)\right| \leqslant|\operatorname{Im}(z)|^{-1}, \quad \sum_{j=1}^{N}\left|R_{i j}(z)\right|^{2} \leqslant\left\|R_{N}(z)\right\|^{2} \leqslant|\operatorname{Im}(z)|^{-2} .
$$

We first prove (3.4) and (3.5). We define the following sets on the complex plane. Let $T$ be an arbitrary large number. Let $L$ be a sufficiently large constant, to be chosen later.

$$
\begin{aligned}
& \mathscr{Q}_{N}:=\left\{z:|z|<T+1 \text { and }|\operatorname{Im}(z)|>L N^{-1 / 5}\right\}, \\
& \mathscr{O}_{N}:=\left\{z:|\operatorname{Im}(z)|>L N^{-1 / 4}\right\} .
\end{aligned}
$$

Note that if $z \in \mathscr{Q}_{N}^{c} \cap\{|z|<T+1\}$, then $|\operatorname{Im}(z)|^{5} \leqslant L^{5} N^{-1}$. When combined with (1.7) this implies

$$
\left|\mathbf{E}\left[R_{i i}(z)\right]-g_{\sigma, c_{N}}(z)\right| \leqslant 2|\operatorname{Im}(z)|^{-1}=O\left(\frac{|\operatorname{Im}(z)|^{-6}}{N}\right) .
$$

Similarly, if $z \in \mathscr{O}_{N}^{c}$, then

$$
\left|\mathbf{E}\left[R_{i k}(z)\right]\right| \leqslant|\operatorname{Im}(z)|^{-1}=O\left(\frac{|\operatorname{Im}(z)|^{-5}}{N}\right) .
$$

For the remainder of the proof of (3.4) and (3.5) we will assume that $z$ is in $\mathscr{Q}_{N}$ and $\mathscr{O}_{N}$, respectively.

The proof of both statements begins with the resolvent identity (3.1), and then an application of the decoupling formula (3.2).

$$
\begin{aligned}
z \mathbf{E}\left[R_{i k}(z)\right]= & \delta_{i k}+\sum_{j=1}^{N} \sum_{l=1}^{n} \mathbf{E}\left[R_{i j}(z) X_{j l} X_{k l}\right] \\
= & \delta_{i k}+\sigma^{2} \mathbf{E}\left[R_{i k}(z) \operatorname{tr}_{N}\left(R_{N}(z) X X^{*}\right)\right] \\
& +\frac{\sigma^{2}}{N} \mathbf{E}\left[\left(R_{N}(z) X X^{*} R(z)\right)_{i k}\right] \\
& +\frac{n \sigma^{2}}{N} \mathbf{E}\left[R_{i k}(z)\right]+r_{N}
\end{aligned}
$$


where $r_{N}$ is the third cumulant term coming from $p=2$ and the error from truncating at $p=2$.

From the definition of the resolvent we have $R_{N}(z)\left(z I_{N}-X X^{*}\right)=I_{N}$, which implies $R_{N}(z) X X^{*}=z R_{N}(z)-I_{N}$. Applying this identity to (3.11) yields

$$
\begin{aligned}
z \mathbf{E}\left[R_{i k}(z)\right]= & \delta_{i k}+\sigma^{2} z \mathbf{E}\left[R_{i k}(z) \operatorname{tr}_{N}\left(R_{N}(z)\right)\right]-\sigma^{2} \mathbf{E}\left[R_{i k}(z)\right] \\
& +\frac{\sigma^{2}}{N} \mathbf{E}\left[\left(R_{N}(z) X X^{*} R_{N}(z)\right)_{i k}\right] \\
& +\frac{n \sigma^{2}}{N} \mathbf{E}\left[R_{i k}(z)\right]+r_{N} .
\end{aligned}
$$

We begin with the following lemma.

Lemma 3.1. For $z \in \mathbb{C} \backslash \mathbf{R}$,

$$
\begin{aligned}
\operatorname{Cov}\left[R_{i j}(z), \operatorname{tr}_{N}\left(R_{N}(z)\right)\right] & \leqslant \frac{P_{2}\left(|\operatorname{Im}(z)|^{-1}\right) \mathbf{E}\left[\left\|R_{N}(z)\right\|^{3 / 2}\right]}{N}, \\
r_{N} & \leqslant \frac{P_{4}\left(|\operatorname{Im}(z)|^{-1}\right)}{N} .
\end{aligned}
$$

Additionally, for $z \in \mathscr{O}_{N}$,

$$
r_{N} \leqslant \frac{P_{2}\left(|\operatorname{Im}(z)|^{-1}\right) \mathbf{E}\left[P_{8}\left(\left\|X_{N}\right\|\right)\left(\left\|R_{N}(z)\right\|^{2}+\left\|R_{N}(z)\right\|^{3 / 2}\right)\right.}{N} .
$$

P r o o f. To prove (3.13) we begin with the following bounds from Proposition 4 in [22]:

$$
\begin{aligned}
& \mathbb{V}\left(\operatorname{tr}_{N}\left(R_{N}(z)\right)\right) \leqslant \frac{|\operatorname{Im}(z)|^{-4}}{N^{2}}, \\
& \mathbb{V}\left(\operatorname{tr}_{N}\left(R_{N}(z)\right)\right) \leqslant \frac{|\operatorname{Im}(z)|^{-7 / 2} \mathbf{E}\left[\left\|R_{N}(z)\right\|\right]^{3 / 2}}{N^{2}} .
\end{aligned}
$$

It follows from the proof of Proposition 4 in [22] that these bounds are valid provided the fourth moments are uniformly bounded ([23]). Additionally, from (3.7) we have

$$
\mathbb{V}\left(R_{i j}(z)\right) \leqslant|\operatorname{Im}(z)|^{-1 / 2} \mathbf{E}\left[\left\|R_{N}(z)\right\|\right]^{3 / 2} .
$$

Using Cauchy-Schwarz this implies

$$
\operatorname{Cov}\left(R_{i j}(z), \operatorname{tr}_{N}\left(R_{N}(z)\right)\right) \leqslant \frac{P_{2}\left(|\operatorname{Im}(z)|^{-1}\right) \mathbf{E}\left[\left\|R_{N}(z)\right\|^{3 / 2}\right]}{N}
$$

as desired. 
Now we prove (3.15); the argument along with Lemma 2.4 can be modified to prove (3.14). The third cumulant term in the decoupling formula is

$$
\begin{aligned}
& \frac{1}{2 N^{3 / 2}} \sum_{j=1}^{N} \sum_{l=1}^{n} \kappa_{3}\left(\left(A_{N}\right)_{k l}\right) \mathbf{E}\left[\frac{\partial^{2} R_{i j}(z) X_{j l}}{\partial X_{k l}^{2}}\right] \\
& =\sum_{j=1}^{N} \sum_{l=1}^{n} \kappa_{3}\left(\left(A_{N}\right)_{k l}\right)\left[R_{i k}(z)\left(X^{*} R(z) X\right)_{l l} R_{k j}(z) X_{j l}\right] \\
& =\frac{1}{N^{3 / 2}} \sum_{l=1}^{n} \kappa_{3}\left(\left(A_{N}\right)_{k l}\right) \mathbf{E}\left[R_{i k}(z)\left(X^{*} R_{N}(z) X\right)_{l l}\left(R_{N}(z) X\right)_{k l}\right. \\
& \quad+\left(R_{N}(z) X\right)_{i l}\left(R_{N}(z) X\right)_{k l}\left(R_{N}(z) X\right)_{k l}+R_{i k}(z)\left(R_{N}(z) X\right)_{k l} \\
& \quad+R_{i k}(z)\left(X^{*} R_{N}(z)\right)_{l k}\left(X^{*} R_{N}(z) X\right)_{l l} \\
& \left.\quad+\left(R_{N}(z) X\right)_{i l} R_{k k}(z)\left(X^{*} R_{N}(z) X\right)_{l l}\right]
\end{aligned}
$$

where $\kappa_{3}\left(\left(A_{N}\right)_{k l}\right)$ is the third cumulant of $\left(A_{N}\right)_{k l}$. By condition C2, the $\kappa_{3}\left(\left(A_{N}\right)_{k l}\right)$ 's are uniformly bounded.

Using (3.7) and the Cauchy-Schwarz inequality this term is seen to be

$$
O\left(\frac{|\operatorname{Im}(z)|^{-1} \mathbf{E}\left[P_{4}\left(\left\|X_{N}\right\|\right)\left\|R_{N}(z)\right\|^{2}\right]}{N}\right) .
$$

The truncation error is bounded from above by a finitely many sums of the following form

$$
\frac{C m_{4}}{N^{2}} \sum_{l=1}^{n} \sup \mathbf{E}\left|R_{a b}^{\prime}(z)\left(R_{N}^{\prime}(z) X^{\prime}\right)_{c d}^{\alpha}\left(R_{N}^{\prime}(z) X^{\prime}\right)_{e f}^{\beta}\left(X^{\prime *} R_{N}^{\prime}(z)\right)_{g h}^{\gamma}\left(X^{\prime *} R_{N}^{\prime} X^{\prime}\right)_{q r}^{\delta}\right|,
$$

where the sup is over all rank two perturbations of $X$ of the form $X^{\prime}=$ $X+x E_{k l}$, where $\left(E_{k l}\right)_{i j}=\delta_{i k} \delta_{j l}+\delta_{i l} \delta_{i j}$ and $R_{N}^{\prime}(z)=\left(z I_{N}-X^{\prime} X^{\prime *}\right)^{-1}$. Additionally, $\alpha+\beta+\gamma+2 \delta \leqslant 4$ and each of $a, b, c, d, e, f, g, h, q, r$ are one of $i, l, k$. The bound (3.14) then immediately follows from (3.7) and Lemma 2.4.

To prove the bound (3.15), we can assume by (iii) of Lemma 2.3 that $|x| \leqslant \varepsilon_{N} N^{-1 / 4}$. Then

$$
\left\|X_{N}^{\prime}\right\|=\left\|X_{N}\right\|+o(1)
$$

Additionally,

$$
R_{N}^{\prime}(z)=R_{N}(z)+R_{N}(z)\left(x E_{k l} X_{N}^{*}+x X_{N} E_{l k}+x^{2} E_{k l} E_{l k}\right) R_{N}^{\prime}(z) .
$$

Thus,

$$
\left\|R_{N}^{\prime}(z)\right\| \leqslant\left\|R_{N}(z)\right\|\left(1+|x| \frac{1}{|\operatorname{Im} z|}\left\|X_{N}\right\|\right) \leqslant\left\|R_{N}(z)\right\|\left(1+\varepsilon_{N}\left\|X_{N}\right\|\right) .
$$

Using (3.19)-(3.21), one obtains (3.15). Lemma 3.1 is proved. 
It follows from (3.12) and Lemma 3.1 that for $i=k$

$$
\left(z-\sigma^{2} z g_{N}(z)-\sigma^{2} c_{N}+\sigma^{2}\right) \mathbf{E} R_{i i}(z)=1+O\left(\frac{P_{4}\left(|\operatorname{Im}(z)|^{-1}\right)}{N}\right) .
$$

Summing over $i$ and dividing by $N$ gives

$$
\left(z-\sigma^{2} z g_{N}(z)-\sigma^{2} c_{N}+\sigma^{2}\right) g_{N}(z)=1+O\left(\frac{P_{4}\left(|\operatorname{Im}(z)|^{-1}\right)}{N}\right) .
$$

Additionally if $i \neq k$

$$
\left(z-\sigma^{2} z g_{N}(z)-\sigma^{2} c_{N}+\sigma^{2}\right) \mathbf{E}\left[R_{i k}(z)\right]=O\left(\frac{P_{4}\left(|\operatorname{Im}(z)|^{-1}\right)}{N}\right) .
$$

Now we use (3.22) and (3.23) and the following lemma to complete the proof of $(3.4)$.

Lemma 3.2. On $\mathscr{Q}_{N},\left|g_{N}(z)-\sigma^{2} g_{N}^{2}(z)\right|$ is uniformly bounded in $z$ and $N$ from below by a positive constant.

$\mathrm{P}$ r o o f. Assume it is not, then for any $\delta>0$ there would exist a $z$ such that $\left|g_{N}(z)-\sigma^{2} g_{N}^{2}(z)\right|<\delta^{2}$ which in turn implies $\left|g_{N}(z)\right|<\delta$ or $\left|\sigma^{2} g_{N}(z)-1\right|<\delta$. But $\left|g_{N}(z)\right|<\delta$ contradicts (3.23) if $\delta$ is sufficiently small once $L$ from $(3.8)$ is chosen to make $O\left(P_{4}\left(|\operatorname{Im}(z)|^{-1}\right) / N\right)$ small enough because $\left(z-\sigma^{2} z g_{N}(z)-\sigma^{2} c_{N}+\sigma^{2}\right)$ is bounded on $\mathscr{Q}_{N}$.

On the other hand, if $\left|\sigma^{2} g_{N}(z)-1\right|<\delta$, then

$$
\begin{aligned}
& \left|\left(z-\sigma^{2} z g_{N}(z)-\sigma^{2} c_{N}+\sigma^{2}\right) g_{N}(z)-1\right| \\
& \quad \geqslant|| z g_{N}(z)-\sigma^{2} z g_{N}^{2}(z)|-| \sigma^{2}\left(1-c_{N}\right) g_{N}(z)-1||,
\end{aligned}
$$

but

$$
\begin{gathered}
\left|z g_{N}(z)-\sigma^{2} z g_{N}^{2}(z)\right|<(T+1) \delta \\
\left|\left(\sigma^{2}\left(1-c_{N}\right) g_{N}(z)-1\right)+c_{N}\right|=\left|\left(1-c_{N}\right)\left(\sigma^{2} g_{N}(z)-1\right)\right| \leqslant\left|\left(1-c_{N}\right) \delta\right| .
\end{gathered}
$$

So for $\delta$ small and $L$ sufficiently large we reach a contradiction with (3.23). Lemma 3.2 is proved.

Let

$$
s_{N}(z):=\frac{1+\sigma^{2} g_{N}(z)\left(c_{N}-1\right)}{g_{N}(z)-\sigma^{2} g_{N}^{2}(z)} .
$$

Then by Lemma 3.2 and (3.22) we have

$$
s_{N}(z)-z=\frac{1+\sigma^{2} g_{N}(z)\left(c_{N}-1\right)}{g_{N}(z)-\sigma^{2} g_{N}^{2}(z)}-z=O\left(\frac{P_{4}\left(|\operatorname{Im}(z)|^{-1}\right)}{N}\right) .
$$

Finally, for $z \in \mathscr{Q}_{N}, g_{N}(z)=g_{\sigma, c_{N}}\left(s_{N}(z)\right)$, which can be seen by evaluating

$$
z \sigma^{2} g_{\sigma, c_{N}}^{2}(z)+\left(\sigma^{2}\left(c_{N}-1\right)-z\right) g_{\sigma, c_{N}}(z)+1=0
$$


at $s_{N}(z)$. This yields

$$
\begin{gathered}
\frac{1+\sigma^{2} g_{N}(z)\left(c_{N}-1\right)}{g_{N}(z)-\sigma^{2} g_{N}^{2}(z)}\left(\sigma^{2} g_{\sigma, c_{N}}^{2}\left(s_{N}(z)\right)-g_{\sigma, c_{N}}\left(s_{N}\right)\right) \\
+\sigma^{2}\left(c_{N}-1\right) g_{\sigma}^{2}\left(s_{N}(z)\right)+1=0 .
\end{gathered}
$$

Rearranging this equation and applying the estimates in (3.7) gives

$$
\left(g_{\sigma, c_{N}}\left(s_{N}(z)\right)-g_{N}(z)\right)\left(\frac{2 \sigma^{2}}{|\operatorname{Im}(z)|^{-1}}+\frac{\sigma^{4}\left(c_{N}-1\right)}{|\operatorname{Im}(z)|^{-2}}\right) \geqslant\left(g_{\sigma, c_{N}}\left(s_{N}(z)\right)-g_{N}(z)\right) .
$$

So $g_{\sigma, c_{N}}\left(s_{N}(z)\right)=g_{N}(z)$ for sufficiently large $z$ and then on $\mathscr{Q}_{N}$ by analytic continuation.

Combining (3.26) and (3.25) gives

$$
\begin{aligned}
\left|g_{N}(z)-g_{\sigma, c_{N}}(z)\right| & =\left|\int \frac{d \mu_{\sigma, c_{N}}(x)}{z-x}-\frac{d \mu_{\sigma, c_{N}}(x)}{s_{N}(z)-x}\right| \\
& \leqslant\left|C\left(s_{N}(z)-z\right) \int \frac{d \mu_{\sigma, c_{N}}(x)}{(z-x)^{2}}\right| \\
& \leqslant \frac{P_{6}\left(|\operatorname{Im}(z)|^{-1}\right)}{N}
\end{aligned}
$$

on $\mathscr{Q}_{N}$. This completes the proof of (3.4).

Beginning from (3.24) we now finish the proof of (3.5). We have

$$
\begin{gathered}
g_{N}(z)\left(z-\sigma^{2} z g_{N}(z)-\sigma^{2} c+\sigma^{2}\right) \mathbf{E}\left[R_{i k}(z)\right]=g_{N}(z)+O\left(\frac{P_{4}\left(|\operatorname{Im}(z)|^{-1}\right)}{N}\right) \\
\left(1+O\left(\frac{P_{4}\left(|\operatorname{Im}(z)|^{-1}\right)}{N}\right)\right) \mathbf{E}\left[R_{i k}(z)\right]=O\left(\frac{P_{5}\left(|\operatorname{Im}(z)|^{-1}\right)}{N}\right) .
\end{gathered}
$$

Recall that $z \in \mathscr{O}_{N}$, and $L$ can be chosen such that $O\left(P_{4}\left(|\operatorname{Im}(z)|^{-1}\right) / N\right)$ on the left-hand side of (3.27) is less than $1 / 2$ in absolute value. Then

$$
\mathbf{E}\left[R_{i k}(z)\right]=O\left(\frac{P_{5}\left(|\operatorname{Im}(z)|^{-1}\right)}{N}\right) .
$$

This completes the proof of (3.5).

Our final step in the proof of Proposition 3.1 is to prove (3.6). First note that if $z \in \mathscr{O}_{N}^{c}$ then

$$
\mathbb{V}\left[R_{i j}(z)\right] \leqslant \mathbf{E}\left[\left\|R_{N}(z)\right\|^{2}\right] \leqslant \frac{L^{4} \mathbf{E}\left[\left\|R_{N}(z)\right\|^{2}\right]}{|\operatorname{Im}(z)|^{4} N} .
$$

For the remainder of the proof we will assume $z \in \mathscr{O}_{N}$. We begin with the resolvent identity (3.1) applied to $\mathbf{E}\left[R_{i k}(z) R_{i k}(\bar{z})\right]$ and then apply the 
decoupling formula (3.2):

$$
\begin{aligned}
z \mathbf{E}[ & \left.R_{i k}(z) R_{i k}(\bar{z})\right]=\delta_{i k} \mathbf{E}\left[R_{i k}(\bar{z})\right]+\sum_{j=1}^{N} \sum_{l=1}^{n} \mathbf{E}\left[\left(R_{i j}(z) R_{i k}(\bar{z}) X_{j l} X_{k l}\right]\right. \\
= & \delta_{i k} \mathbf{E}\left[R_{i k}(\bar{z})\right]+\sigma^{2} \mathbf{E}\left[R_{i k}(z) R_{i k}(\bar{z}) \operatorname{tr}_{N}\left(X^{*} R_{N}(z) X\right)\right] \\
& +\frac{\sigma^{2}}{N} \mathbf{E}\left[\left(R_{N}(z) X X^{*} R_{N}(z)\right)_{i k} R_{i k}(\bar{z})\right] \\
& +\frac{\sigma^{2}}{N} \mathbf{E}\left[\left(R_{i k}(\bar{z})\left(R_{N}(z) X X^{*} R_{N}(\bar{z})\right)_{i k}+\left(R_{N}(\bar{z}) X X^{*} R_{N}(z)\right)_{i i} R_{k k}(\bar{z})\right)\right] \\
& +\frac{\sigma^{2} n}{N} \mathbf{E}\left[R_{i k}(z) R_{i k}(\bar{z})\right]+r_{N},
\end{aligned}
$$

where $r_{N}$ contains the third cumulant term, $p=2$, and the error for truncating at $p=2$.

Once again using that $R_{N}(z) X X^{*}=z R_{N}(z)-I_{N}$ gives

$$
\begin{array}{rl}
z & \mathbf{E}\left[R_{i k}(z) R_{i k}(\bar{z})\right]=\delta_{i k} \mathbf{E}\left[R_{i k}(\bar{z})\right]+\sigma^{2} z \mathbf{E}\left[R_{i k}(z) R_{i k}(\bar{z}) \operatorname{tr}_{N}\left(R_{N}(z)\right)\right] \\
& -\sigma^{2} \mathbf{E}\left[R_{i k}(z) R_{i k}(\bar{z})\right]+\frac{\sigma^{2}}{N} \mathbf{E}\left[\left(R_{N}(z) X X^{*} R_{N}(z)\right)_{i k} R_{i k}(\bar{z})\right] \\
& +\frac{\sigma^{2}}{N} \mathbf{E}\left[\left(R_{i k}(\bar{z})\left(R_{N}(z) X X^{*} R_{N}(\bar{z})\right)_{i k}+\left(R_{N}(\bar{z}) X X^{*} R_{N}(z)\right)_{i i} R_{k k}(\bar{z})\right)\right] \\
& +\frac{\sigma^{2} n}{N} \mathbf{E}\left[R_{i k}(z) R_{i k}(\bar{z})\right]+r_{N} .
\end{array}
$$

Similar to Lemma 3.1 we use the following lemma to complete our variance bound.

Lemma 3.3. For $z \in \mathbb{C} \backslash \mathbf{R}$ :

$$
\begin{gathered}
\operatorname{Cov}\left[R_{i j}(z) R_{i k}(\bar{z}), \operatorname{tr}_{N}\left(R_{N}(z)\right)\right] \leqslant \frac{P_{3}\left(|\operatorname{Im}(z)|^{-1}\right) \mathbf{E}\left[\left\|R_{N}(z)\right\|^{3 / 2}\right]}{N}, \\
r_{N} \leqslant \frac{P_{3}\left(|\operatorname{Im}(z)|^{-1}\right) \mathbf{E}\left[P_{10}\left(\left\|X_{N}\right\|\right)\left(\left\|R_{N}(z)\right\|^{2}+\left\|R_{N}(z)\right\|^{3 / 2}\right)\right]}{N} .
\end{gathered}
$$

P r o o f. The proof follows from the steps taken in the proof of Lemma 3.1. For the reader's convenience the third cumulant term is

$$
\begin{aligned}
& \frac{1}{2 N^{3 / 2}} \sum_{j=1}^{N} \sum_{l=1}^{n} \kappa_{3}\left(\left(A_{N}\right)_{k l}\right) \mathbf{E}[\left.\frac{\partial^{2} R_{i j}(z) X_{j l} R_{i k}(\bar{z})}{\partial X_{k l}^{2}}\right] \\
&=\frac{1}{2 N^{3 / 2}} \sum_{l=1}^{n} \kappa_{3}\left(\left(A_{N}\right)_{k l}\right) \mathbf{E}\left[\frac{\partial^{2}\left(R_{N}(z) X\right)_{i l}}{\partial^{2} X_{k l}} R_{i k}(\bar{z})\right. \\
&+2 \frac{\partial\left(R_{N}(z) X\right)_{i l}}{\partial X_{k l}} \frac{\partial R_{i k}(\bar{z})}{\partial X_{k l}} \\
&\left.+\left(R_{N}(z) X\right)_{i l} \frac{\partial^{2} R_{i k}(\bar{z})}{\partial^{2} X_{k l}}\right] .
\end{aligned}
$$


The first subsum is

$$
\begin{aligned}
\frac{1}{2 N^{3 / 2}} \sum_{l=1}^{n} & \kappa_{3}\left(\left(A_{N}\right)_{k l}\right)\left(R_{i k}(z)\left(X^{*} R_{N}(z) X\right)_{l l}\left(R_{N}(z) X_{k l}\right) R_{i k}(\bar{z})\right. \\
& +2(R(z) X)_{i l}\left(R_{N}(z) X\right)_{k l}\left(R_{N}(z) X\right)_{k l} R_{i k}(\bar{z}) \\
& +2 R_{i k}(z)\left(R_{N}(z) X\right)_{k l} R_{i k}(\bar{z}) \\
& +2 R_{i k}(z)\left(X^{*} R_{N}(z)\right)_{l k}\left(X^{*} R_{N}(z) X\right)_{l l} R_{i k}(\bar{z}) \\
& \left.+2\left(R_{N}(z) X\right)_{i l} R_{k k}(z)\left(X^{*} R_{N}(z) X\right)_{l l} R_{i k}(\bar{z})\right)
\end{aligned}
$$

The second subsum is

$$
\begin{aligned}
\frac{1}{2 N^{3 / 2}} & \sum_{l=1}^{n} \kappa_{3}\left(\left(A_{N}\right)_{k l}\right)\left(2 R_{i k}(z)\left(X^{*} R_{N}(z) X\right)_{l l} R_{i k}(\bar{z})\left(X^{*} R_{N}(\bar{z})\right)_{l k}\right. \\
& +2\left(R_{N}(z) X\right)_{i l}\left(R_{N}(z) X\right)_{k l} R_{i k}(\bar{z})\left(X^{*} R_{N}(\bar{z})\right)_{l k} \\
& +2 R_{i k}(z)\left(X^{*} R_{N}(z) X\right)_{l l}\left(R_{N}(\bar{z}) X\right)_{i l} R_{k k}(\bar{z}) \\
& +2\left(R_{N}(z) X\right)_{i l}(z)\left(R_{N}(z) X\right)_{k l}\left(R_{N}(\bar{z}) X\right)_{i l} R_{k k}(\bar{z}) \\
& \left.+2 R_{i k}(z) R_{i k}(\bar{z})\left(X^{*} R_{N}(\bar{z})\right)_{l k}+2 R_{i k}(z)\left(R_{N}(\bar{z}) X\right)_{i l} R_{k k}(\bar{z})\right) .
\end{aligned}
$$

The third subsum is

$$
\begin{aligned}
\frac{1}{2 N^{3 / 2}} \sum_{l=1}^{n} \kappa_{3} & \left(\left(A_{N}\right)_{k l}\right)\left(2\left(R_{N}(z) X\right)_{i l} R_{i k}(\bar{z})\left(X^{*} R_{N}(\bar{z}) X\right)_{l l} R_{k k}(\bar{z})\right. \\
& +2\left(R_{N}(z) X\right)_{i l}\left(R_{N}(\bar{z}) X\right)_{i l}\left(R_{N}(\bar{z}) X\right)_{k l} R_{k k}(\bar{z}) \\
& +2\left(R_{N}(z) X\right)_{i l} R_{i k}(\bar{z}) R_{k k}(\bar{z}) \\
& +2\left(R_{N}(z) X\right)_{i l} R_{i k}(\bar{z})\left(X^{*} R_{N}(\bar{z})\right)_{l k}\left(X^{*} R_{N}(\bar{z})\right)_{l k} \\
& \left.+2\left(R_{N}(z) X\right)_{i l}\left(R_{N}(\bar{z}) X\right)_{i l} R_{k k}(\bar{z})\left(X^{*} R_{N}(\bar{z})\right)_{l k}\right)
\end{aligned}
$$

Once again by (3.7) and the Cauchy-Schwarz inequality this term is bounded by

$$
O\left(\frac{P_{2}\left(|\operatorname{Im}(z)|^{-1}\right) \mathbf{E}\left[P_{4}\left(\left\|X_{N}\right\|\right)\left\|R_{N}(z)\right\|^{2}\right]}{N}\right) .
$$

The error term due to the truncation of the decoupling formula at $p=2$ is estimated as in (3.19)-(3.21) in Lemma 3.1. Lemma 3.3 is proved.

Then using (3.12) to subtract $\mathbf{E}\left[R_{i k}(z)\right] \mathbf{E}\left[R_{i k}(\bar{z})\right]$ from (3.28) gives

$$
\begin{gathered}
\left(z-\sigma^{2} z g_{N}(z)+\sigma^{2}\left(1-c_{N}\right)\right)\left(\mathbf{E}\left[R_{i k}(z) R_{i k}(\bar{z})\right]-\mathbf{E}\left[R_{i k}(z)\right] \mathbf{E}\left[R_{i k}(\bar{z})\right]\right) \\
=O\left(\frac{P_{3}\left(|\operatorname{Im}(z)|^{-1}\right)\left(\mathbf { E } \left[P _ { 1 0 } \left(\|\left(X_{N} \|\right)\left(\left\|R_{N}(z)\right\|^{2}+\left\|R_{N}(z)\right\|^{3 / 2}\right)\right.\right.\right.}{N}\right) .
\end{gathered}
$$

Repeating the argument for (3.5) leads to

$$
\mathbb{V}\left(R_{i k}(z)\right)=O\left(\frac{P_{4}\left(|\operatorname{Im}(z)|^{-1}\right)\left(\mathbf { E } \left[P _ { 1 0 } \left(\|\left(X_{N} \|\right)\left(\left\|R_{N}(z)\right\|^{2}+\left\|R_{N}(z)\right\|^{3 / 2}\right)\right.\right.\right.}{N}\right) .
$$

Lemma 3.3 is proved. 
4. Functional calculus. We now extend the results of Section 3 from resolvents to a more general class of functions. To do this we use the HelfferSjöstrand functional calculus ([10], [7]). Let $f \in C^{l+1}(\mathbf{R})$, functions with $l+1$ continuous derivatives that decay at infinity sufficiently fast. Then one can write

$$
f\left(X_{N}\right)=-\frac{1}{\pi} \int_{\mathbf{C}} \frac{\partial \tilde{f}}{\partial \bar{z}} R_{N}(z) d x d y, \quad \frac{\partial \tilde{f}}{\partial \bar{z}}:=\frac{1}{2}\left(\frac{\partial \tilde{f}}{\partial x}+i \frac{\partial \tilde{f}}{\partial y}\right)
$$

where

(i) $z=x+i y$ with $x, y \in \mathbf{R}$;

(ii) $\tilde{f}(z)$ is the extension of the function $f$ defined as follows

$$
\tilde{f}(z):=\left(\sum_{n=0}^{l} \frac{f^{(n)}(x)(i y)^{n}}{n !}\right) \sigma(y)
$$

here $\sigma \in C^{\infty}(\mathbf{R})$ is a nonnegative function equal to 1 for $|y| \leqslant 1 / 2$ and equal to zero for $|y| \geqslant 1$.

From its definition one can see that (4.2) satisfies the following bound:

$$
\left|\frac{\partial \tilde{f}}{\partial \bar{z}}(x+i y)\right| \leqslant \text { Const } \max \left(\left|\frac{d^{j} f}{d x^{j}}(x)\right|, 0 \leqslant j \leqslant l+1\right)|y|^{l} .
$$

Proposition 4.1. Let $A_{N}$ be an $N \times n$ real (complex) matrix that satisfies condition $\mathbf{C 2}(\mathbf{C 1})$. Let $M_{N}=A_{N} A_{N}^{*} / N$.

(i) Let $f: \mathbf{R} \rightarrow \mathbf{R}$ such that $\operatorname{supp}(f) \cap \mathbf{R}_{+} \subset[0, L]$ for some $L>0$ and $\|f\|_{C^{7}([0, L])}<\infty$, then there exists a constant $C\left(L, \sigma, m_{4}\right)$ such that $\left|\mathbf{E}\left[f\left(M_{N}\right)_{i i}\right]-\int f(x) d \mu_{\sigma, c_{N}}(x)\right| \leqslant C\left(L, \sigma, m_{4}\right)\|f\|_{C^{7}([0, L])} N^{-1} \quad$ for $1 \leqslant i \leqslant N$.

(ii) Let $f: \mathbf{R} \rightarrow \mathbf{R}$ such that $\|f\|_{C^{6}\left(\mathbf{R}_{+}\right)}<\infty$, then there exists a constant $C\left(\sigma, m_{4}\right)$ such that

$$
\left|\mathbf{E}\left[f\left(M_{N}\right)_{i k}\right]\right| \leqslant C\left(\sigma, m_{4}\right)\|f\|_{C^{6}\left(\mathbf{R}_{+}\right)} N^{-1} \quad \text { for } 1 \leqslant i \neq k \leqslant N .
$$

(iii) Let $f: \mathbf{R} \rightarrow \mathbf{R}$ such $\|f\|_{s}<\infty$, for $s>3$, then there exists a constant $C\left(s, \sigma, m_{4}\right)$ such that

$$
\left|\mathbb{V}\left[f\left(M_{N}\right)_{i j}\right]\right| \leqslant C\left(s, \sigma, m_{4}\right)\|f\|_{s} N^{-1} \quad \text { for } 1 \leqslant i, j \leqslant N .
$$

The proof follows as in [17]. We sketch the details below.

$\mathrm{P}$ r o o f. First, we note that since $M_{N}$ is a nonnegative definite matrix, changing the values of $f(x)$ for negative $x$ does not have any effect on the matrix values $f\left(M_{N}\right)_{i j}$. For example, we can always multiply $f$ by a smooth 
function $\varphi$ which is identically 1 on $R_{+}$and 0 on $(-\infty,-\delta]$. By the HelfferSjöstrand functional calculus we have

$$
\begin{aligned}
\mathbf{E}\left[f\left(M_{N}\right)_{i i}\right] & =\mathbf{E}\left[-\frac{1}{\pi} \int_{\mathbb{C}} \frac{\partial \tilde{f}}{\partial \bar{z}} R_{i i}(z) d x d y\right] \\
& =\mathbf{E}\left[-\frac{1}{\pi} \int_{\mathbb{C}} \frac{\partial \tilde{f}}{\partial \bar{z}}\left(g_{\sigma}(z)+\varepsilon_{i i}\right) d x d y\right] \\
& =\int f(x) d \mu_{\sigma, c_{N}}(x)-\mathbf{E}\left[\frac{1}{\pi} \int_{\mathbb{C}} \frac{\partial \tilde{f}}{\partial \bar{z}} \varepsilon_{i i} d x d y\right]
\end{aligned}
$$

where

$$
\left|\varepsilon_{i i}\right|=\mid \mathbf{E}\left[R_{i i}(z)-g_{\sigma, c_{N}}(z)|\leqslant| \frac{P_{6}\left(|\operatorname{Im}(z)|^{-1}\right)}{N} \mid\right.
$$

by (3.4). Combining this inequality with (4.3), letting $l=6$ yields

$$
\mathbf{E}\left[\frac{1}{\pi} \int_{\mathbf{C}} \frac{\partial \tilde{f}}{\partial \bar{z}} \varepsilon_{i i} d x d y\right] \leqslant C\|f\|_{C^{7}([0, L])} N^{-1} .
$$

Completing the proof of (4.4). The proof of (4.5) follows similarly.

The rest of the proof of (4.6) follows the proof of Proposition 4.2 in [17], using Proposition 1 from [22].

We first consider the diagonal case, $i=j$, without loss of generality let $i=1$ and define the random spectral measure

$$
\mu(d x, \omega):=\sum_{l=1}^{N} \delta\left(x-\lambda_{l}\right)\left|\phi_{l}(1)\right|^{2}
$$

Where $\lambda_{l}$ are the eigenvalues of $M_{N}$ and $\phi_{l}$ are the corresponding normalized eigenvectors.

Proposition 2.2 of [17] applied to the measure (4.7) gives

$$
\mathbb{V}\left[f\left(M_{N}\right)_{11}\right] \leqslant C_{s}\|f\|_{s}^{2} \int_{0}^{\infty} d y e^{-y} y^{2 s-1} \int_{-\infty}^{\infty} \mathbb{V}\left[R_{11}(x+i y)\right] d x .
$$

Using (3.6) we can estimate $\int_{-\infty}^{\infty} \mathbb{V}\left[R_{i j}(x+i y)\right] d x$ from above by

$$
\frac{P_{4}\left(y^{-1}\right)}{N} \mathbf{E}\left[\int_{-\infty}^{\infty} P_{10}\left(\left\|X_{N}\right\|\right)\left(\left\|R_{N}(x+i y)\right\|^{2}+\left\|R_{N}(x+i y)\right\|^{3 / 2}\right) d x\right] .
$$

Once we open the brackets, we obtain two terms. Here, we bound the first term. The other term can be estimated in a similar way:

$$
\begin{gathered}
\mathbf{E}\left[\int_{-\infty}^{\infty} P_{10}\left(\left\|X_{N}\right\|\right)\left\|R_{N}(x+i y)\right\|^{2} d x\right] \\
\leqslant \mathbf{E}\left[P _ { 1 0 } ( \| X _ { N } \| ) \left(\int_{-\left\|X_{N} X_{N}^{*}\right\|}^{\left\|X_{N} X_{N}^{*}\right\|} y^{-2} d x\right.\right.
\end{gathered}
$$




$$
\begin{gathered}
\left.\left.+\int_{|x|>\left\|X_{N} X_{N}^{*}\right\|} \frac{1}{\left(x-\left\|X_{N} X_{N}^{*}\right\|\right)^{2}+y^{2}} d x\right)\right] \\
\leqslant P_{2}\left(y^{-1}\right) \mathbf{E} P_{12}\left(\left\|X_{N}\right\|\right) .
\end{gathered}
$$

By Lemma $2.4(4.8)$ can be bounded by $C P_{2}\left(y^{-1}\right)$. This leads to

$$
\mathbb{V}\left[f\left(X_{N}\right)_{11}\right] \leqslant C_{s} \frac{\|f\|_{s}^{2}}{N} \int_{0}^{\infty} d y e^{-y} y^{2 s-1} P_{6}\left(y^{-1}\right) .
$$

The integral converges if $s>3$.

In the off-diagonal case $i \neq j$, we consider the (complex-valued) measure

$$
\mu(d x, \omega):=\sum_{l=1}^{N} \delta\left(x-\lambda_{l}\right) \overline{\phi_{l}(i)} \phi_{l}(j),
$$

which is a linear combination of probability measures, and apply Proposition 2.2 of [17] to each probability measure in the linear combination. Proposition 4.1 is proven.

5. Resolvent CLT. Let $m$ be a fixed positive integer and let $R_{N}^{(m)}(z)$ denote the $m \times m$ upper-left corner of the resolvent matrix, $R_{N}(z)$. Define

$$
\Psi_{N}(z)=\sqrt{N}\left(R_{N}^{(m)}(z)-g_{\sigma, c_{N}}(z) I\right), \quad z \in \mathbb{C} \backslash\left[0, \sigma^{2}(1+\sqrt{c})^{2}\right] .
$$

Clearly, $\Psi_{N}(z)$ is well defined for $z \in \mathbb{C} \backslash \mathbf{R}$. By Lemma 2.2, $\Psi_{N}(z)$ is well defined for $z \in \mathbf{R} \backslash\left[0, \sigma^{2}(1+\sqrt{c})^{2}\right]$ with probability going to 1 . We are interested in studying the random function $\Psi_{N}(z)$ whose values are in the space of complex symmetric $m \times m$ matrices. We also define

$$
\varphi(z, w)=\mathbf{E}\left[\frac{z}{z-\eta_{1 / c}} \frac{w}{w-\eta_{1 / c}}\right],
$$

where $\eta_{1 / c}$ is a Marchenko-Pastur distributed random variable with ratio index $1 / c$ and scale index $\sigma^{2}$. We introduce the following notation:

$$
\begin{aligned}
& \varphi_{++}(z, w)=\mathbf{E}\left[\operatorname{Re} \frac{z}{z-\eta_{1 / c}} \operatorname{Re} \frac{w}{w-\eta_{1 / c}}\right], \\
& \varphi_{--}(z, w)=\mathbf{E}\left[\operatorname{Im} \frac{z}{z-\eta_{1 / c}} \operatorname{Im} \frac{w}{w-\eta_{1 / c}}\right], \\
& \varphi_{+-}(z, w)=\mathbf{E}\left[\operatorname{Re} \frac{z}{z-\eta_{1 / c}} \operatorname{Im} \frac{w}{w-\eta_{1 / c}}\right] .
\end{aligned}
$$

Theorem 5.1. Let $A_{N}$ be a $N \times n$ random matrix with real entries that satisfies condition $\mathbf{C 2}$. Let $m$ be a fixed positive integer and assume that for $1 \leqslant i \leqslant m$

$$
m_{4}(i):=\lim _{N \rightarrow \infty} \frac{1}{n} \sum_{j} \mathbf{E}\left|A_{i j}\right|^{4}
$$


exists and for all $\varepsilon>0$ (1.11) holds. Let

$$
\kappa_{4}(i):=m_{4}(i)-3 \sigma^{4}, \quad 1 \leqslant i \leqslant m .
$$

Then the random field $\Psi_{N}(z)$ converges in finite-dimensional distributions to the random field $\Psi(z)=\sqrt{c} g_{\sigma, c}^{2}(z) Y(z)$, where $Y(z)=\left(Y_{i j}(z)\right)_{1 \leqslant i, j \leqslant m}$ is the Gaussian random field such that the entries $Y_{i j}(z), i \leqslant j$, and $Y_{k l}(w)$, $k \leqslant l$, are independent when $(i, j) \neq(k, l)$ and

$$
\begin{aligned}
\operatorname{Cov}\left(\operatorname{Re} Y_{i i}(c z), \operatorname{Re} Y_{i i}(c w)\right)= & \kappa_{4}(i) \operatorname{Re}\left[z g_{\sigma, 1 / c}(z)\right] \operatorname{Re}\left[w g_{\sigma, 1 / c}(w)\right] \\
& +2 \sigma^{4} \varphi_{++}(z, w), \\
\operatorname{Cov}\left(\operatorname{Im} Y_{i i}(c z), \operatorname{Im} Y_{i i}(c w)\right)= & \kappa_{4}(i) \operatorname{Im}\left[z g_{\sigma, 1 / c}(z)\right] \operatorname{Im}\left[w g_{\sigma, 1 / c}(w)\right] \\
& +2 \sigma^{4} \varphi_{--}(z, w), \\
\operatorname{Cov}\left(\operatorname{Re} Y_{i i}(c z), \operatorname{Im} Y_{i i}(c w)\right)= & \kappa_{4}(i) \operatorname{Re}\left[z g_{\sigma, 1 / c}(z)\right] \operatorname{Im}\left[w g_{\sigma, 1 / c}(w)\right] \\
& +2 \sigma^{4} \varphi_{+-}(z, w), \\
\operatorname{Cov}\left(\operatorname{Re} Y_{i j}(c z), \operatorname{Re} Y_{i j}(c w)\right)= & \sigma^{4} \varphi_{++}(z, w), \quad i \neq j \\
\operatorname{Cov}\left(\operatorname{Im} Y_{i j}(c z), \operatorname{Im} Y_{i j}(c w)\right)= & \sigma^{4} \varphi_{--}(z, w), \quad i \neq j, \\
\operatorname{Cov}\left(\operatorname{Re} Y_{i j}(c z), \operatorname{Im} Y_{i j}(c w)\right)= & \sigma^{4} \varphi_{+-}(z, w), \quad i \neq j
\end{aligned}
$$

In the Hermitian case we have the following theorem.

Theorem 5.2. Let $A_{N}$ be a $N \times n$ random matrix with complex entries that satisfies condition $\mathbf{C 1}$. Let $m$ be a fixed positive integer and assume that for $1 \leqslant i \leqslant m$

$$
m_{4}(i):=\lim _{N \rightarrow \infty} \frac{1}{n} \sum_{j} \mathbf{E}\left|A_{i j}\right|^{4}
$$

exists and for all $\varepsilon>0$ (1.11) holds. Let

$$
\kappa_{4}(i):=m_{4}(i)-2 \sigma^{4}, \quad 1 \leqslant i \leqslant m .
$$

Then the random field $\Psi_{N}(z)$ converges in finite-dimensional distributions to the random field $\Psi(z)=\sqrt{c} g_{\sigma, c}^{2}(z) Y(z)$, where $Y(z)=\left(Y_{i j}(z)\right)_{1 \leqslant i, j \leqslant m}$ is the Gaussian random field such that the entries $Y_{i j}(z), i \leqslant j$, and $Y_{k l}(w)$, $k \leqslant l$, are independent, when $(i, j) \neq(k, l)$ and

$$
\begin{aligned}
\operatorname{Cov}\left(\operatorname{Re} Y_{i i}(c z), \operatorname{Re} Y_{i i}(c w)\right)= & \kappa_{4}(i) \operatorname{Re}\left[z g_{\sigma, 1 / c}(z)\right] \operatorname{Re}\left[w g_{\sigma, 1 / c}(w)\right] \\
& +\sigma^{4} \varphi_{++}(z, w), \\
\operatorname{Cov}\left(\operatorname{Im} Y_{i i}(c z), \operatorname{Im} Y_{i i}(c w)\right)= & \kappa_{4}(i) \operatorname{Im}\left[z g_{\sigma, 1 / c}(z)\right] \operatorname{Im}\left[w g_{\sigma, 1 / c}(w)\right] \\
& +\sigma^{4} \varphi_{--}(z, w), \\
\operatorname{Cov}\left(\operatorname{Re} Y_{i i}(c z), \operatorname{Im} Y_{i i}(c w)\right)= & \kappa_{4}(i) \operatorname{Re}\left[z g_{\sigma, 1 / c}(z)\right] \operatorname{Im}\left[w g_{\sigma, 1 / c}(w)\right] \\
& +\sigma^{4} \varphi_{+-}(z, w), \\
\operatorname{Cov}\left(\operatorname{Re} Y_{i j}(c z), \operatorname{Re} Y_{i j}(c w)\right)= & \frac{1}{2} \sigma^{4}\left(\varphi_{++}(z, w)+\varphi_{--}(z, w)\right), \quad i \neq j,
\end{aligned}
$$




$$
\begin{array}{ll}
\operatorname{Cov}\left(\operatorname{Im} Y_{i j}(c z), \operatorname{Im} Y_{i j}(c w)\right)=\frac{1}{2} \sigma^{4}\left(\varphi_{++}(z, w)+\varphi_{--}(z, w)\right), & i \neq j, \\
\operatorname{Cov}\left(\operatorname{Re} Y_{i j}(c z), \operatorname{Im} Y_{i j}(c w)\right)=\frac{1}{2} \sigma^{4}\left(\varphi_{+-}(z, w)-\varphi_{+-}(w, z)\right), & i \neq j .
\end{array}
$$

$\mathrm{R}$ e $\mathrm{m}$ a $\mathrm{rk}$ 5.1. We remind the reader that the covariance values in Theorems 5.1 and 5.2 are stated in terms of the Marchenko-Pastur law with ratio index $1 / c$ and scale index $\sigma^{2}$. In some cases it may be more convenient to state the covariances in terms of the Marchenko-Pastur law with ratio index $c$. Indeed, a simple computation reveals that for $c>0$ and any continuous function $f$,

$$
\mathbf{E}\left[f\left(\eta_{1 / c}\right)\right]=\frac{1}{c} \mathbf{E}\left[f\left(\frac{\eta_{c}}{c}\right)\right]+\left(1-\frac{1}{c}\right) f(0),
$$

where $\eta_{c}$ is a Marchenko-Pastur distributed random variable with ratio index $c$ and scale index $\sigma^{2}$. In particular, we note that

$$
\begin{gathered}
g_{\sigma, 1 / c}(z)=g_{\sigma, c}(c z)+\left(1-\frac{1}{c}\right) \frac{1}{z}, \\
\varphi(z, w)=\frac{1}{c} \mathbf{E}\left[\frac{c z}{c z-\eta_{c}} \frac{c w}{c w-\eta_{c}}\right]+\left(1-\frac{1}{c}\right) .
\end{gathered}
$$

We will need the following lemma for the proof of Theorems 5.1 and 5.2.

Lemma 5.1. Let $B$ be an $N \times n$ matrix. Then

$$
B^{*}\left(z-B B^{*}\right)^{-1} B=B^{*} B\left(z-B^{*} B\right)^{-1}
$$

for all $z \notin \operatorname{Sp}\left(B B^{*}\right) \cup\{0\}$.

P r o o f. Choose $z \notin \operatorname{Sp}\left(B B^{*}\right) \cup\{0\}$ such that $|z|>\left\|B B^{*}\right\|$. Then we have that

$$
\begin{aligned}
B^{*}\left(z-B B^{*}\right)^{-1} B & =B^{*} \frac{1}{z}\left(I+\sum_{k=1}^{\infty} \frac{1}{z^{k}}\left(B B^{*}\right)^{k}\right) B \\
& =B^{*} B \frac{1}{z}\left(I+\sum_{i=1}^{\infty} \frac{1}{z^{k}}\left(B^{*} B\right)^{k}\right) \\
& =B^{*} B\left(z-B^{*} B\right)^{-1} .
\end{aligned}
$$

We can now extend the result to all $z \notin \operatorname{Sp}\left(B B^{*}\right) \cup\{0\}$ by analytic continuation of the function

$$
f_{u v}(z)=\left\langle B^{*}\left(z-B B^{*}\right)^{-1} B u, v\right\rangle,
$$

where $u, v$ are arbitrary vectors. Lemma 5.1 is proved.

We present the proof of Theorem 5.1 below. The proof in the Hermitian case is similar (see also [19] and [17]) and is left to the reader. 
Pro of of $\mathrm{Th}$ e or e $\mathrm{m}$ 5.1. We write

$$
A_{N}=\left(\begin{array}{c}
r_{1} \\
r_{2} \\
\vdots \\
r_{N}
\end{array}\right), \quad A_{N}^{(m)}=\left(\begin{array}{c}
r_{m+1} \\
r_{m+2} \\
\vdots \\
r_{N}
\end{array}\right)
$$

where $r_{i}$ is an $n$-vector representing the $i$-th row of $A_{N}$. We remind the reader that $X_{N}=A_{N} / \sqrt{N}$ and we will use the notation $X_{N}^{(m)}=A_{N}^{(m)} / \sqrt{N}$. Recall that we denote by $R_{N}^{(m)}(z)$ the $m \times m$ upper-left corner of the resolvent matrix, $R_{N}(z)$, of $M_{N}=A_{N} A_{N}^{*} / N=X_{N} X_{N}^{*}$.

Standard linear algebra gives (see, e.g., [17])

$$
R_{N}^{(m)}(z)=\left(z I_{m}-\left(\frac{1}{N} r_{i} B_{N}^{(m)}(z) r_{j}^{*}\right)_{i, j=1}^{m}\right)^{-1},
$$

where

$$
B_{N}^{(m)}(z)=I_{n}+X_{N}^{(m)^{*}}\left(z-X_{N}^{(m)} X_{N}^{(m)^{*}}\right)^{-1} X_{N}^{(m)}
$$

Let

$$
\Gamma_{N}(z)=\sqrt{N}\left[\left(\frac{1}{N} r_{i} B_{N}^{(m)}(z) r_{j}^{*}\right)_{i, j=1}^{m}-\sigma^{2}\left(c_{N}-1+z g_{\sigma, c_{N}}\right) I_{m}\right] .
$$

Then a simple computation reveals that

$$
R_{N}^{(m)}=\left[\frac{1}{g_{\sigma, c_{N}}(z)} I_{m}-\frac{1}{\sqrt{N}} \Gamma_{N}(z)\right]^{-1} .
$$

It will follow from the central limit theorem for quadratic forms (see the appendix of [17]), that $\left\|\Gamma_{N}(z)\right\|$ is bounded in probability for $z \in \mathbb{C} \backslash \mathbf{R}$. Thus, we have that

$$
\Psi_{N}(z)=g_{\sigma, c_{N}}^{2}(z) \Gamma_{N}(z)+o(1) .
$$

We note that by (5.5),

$$
\frac{1}{N} \operatorname{Tr} B_{N}^{(m)}(z)=c_{N}-1+\frac{z}{N} \operatorname{Tr}\left(z-X_{N}^{(m)} X_{N}^{(m)^{*}}\right)^{-1}
$$

and

$$
\sqrt{N}\left[\frac{1}{N} \operatorname{Tr}\left(z-X_{N}^{(m)} X_{N}^{(m)^{*}}\right)^{-1}-g_{\sigma, c}(z)\right] \longrightarrow 0
$$

in probability as $N \rightarrow \infty$ by (3.16).

Thus, we have

$$
\begin{aligned}
\Gamma_{N}(z) & =\sqrt{N}\left[\left(\frac{1}{N} r_{i} B_{N}^{(m)}(z) r_{j}^{*}\right)_{i, j=1}^{m}-\frac{\sigma^{2}}{N} \operatorname{Tr} B_{N}^{(m)}(z) I_{m}\right]+o(1) \\
& =\sqrt{c_{N} n}\left[\left(\frac{1}{n} r_{i} B_{N}^{(m)}(z) r_{j}^{*}\right)_{i, j=1}^{m}-\frac{\sigma^{2}}{n} \operatorname{Tr} B_{N}^{(m)}(z) I_{m}\right]+o(1) .
\end{aligned}
$$


By Lemma 5.1 and (5.5), we have

$$
\begin{aligned}
B_{N}^{(m)}(z) & =z\left(z-X_{N}^{(m)^{*}} X_{N}^{(m)}\right)^{-1} \\
& =\frac{z}{c_{N}}\left(\frac{z}{c_{N}}-\frac{1}{n} A_{N}^{(m)^{*}} A_{N}^{(m)}\right)^{-1} .
\end{aligned}
$$

By Theorem 1.1 and (5.6), we note that

$$
\begin{aligned}
& \frac{1}{n} \operatorname{Tr}\left[\operatorname{Re} B_{N}^{(m)}(z) \operatorname{Re} B_{N}^{(m)}(w)\right] \longrightarrow \varphi_{++}\left(\frac{z}{c}, \frac{w}{c}\right), \\
& \frac{1}{n} \operatorname{Tr}\left[\operatorname{Im} B_{N}^{(m)}(z) \operatorname{Im} B_{N}^{(m)}(w)\right] \longrightarrow \varphi_{--}\left(\frac{z}{c}, \frac{w}{c}\right), \\
& \frac{1}{n} \operatorname{Tr}\left[\operatorname{Re} B_{N}^{(m)}(z) \operatorname{Im} B_{N}^{(m)}(w)\right] \longrightarrow \varphi_{+-}\left(\frac{z}{c}, \frac{w}{c}\right),
\end{aligned}
$$

in probability as $N \rightarrow \infty$. We now claim that for $1 \leqslant i \leqslant m$,

$$
\begin{aligned}
& \frac{1}{n} \sum_{j=1}^{n} \kappa_{4}\left(A_{N}\right)_{i j} {\left[\operatorname{Re} B_{N}^{(m)}(z)\right]_{j j}\left[\operatorname{Re} B_{N}^{(m)}(w)\right]_{j j} } \\
& \longrightarrow \kappa_{4}(i) \operatorname{Re}\left[\frac{z}{c} g_{\sigma, 1 / c}\left(\frac{z}{c}\right)\right] \operatorname{Re}\left[\frac{w}{c} g_{\sigma, 1 / c}\left(\frac{w}{c}\right)\right], \\
& \frac{1}{n} \sum_{j=1}^{n} \kappa_{4}\left(A_{N}\right)_{i j}\left[\operatorname{Im} B_{N}^{(m)}(z)\right]_{j j}\left[\operatorname{Im} B_{N}^{(m)}(w)\right]_{j j} \\
& \longrightarrow \kappa_{4}(i) \operatorname{Im}\left[\frac{z}{c} g_{\sigma, 1 / c}\left(\frac{z}{c}\right)\right] \operatorname{Im}\left[\frac{w}{c} g_{\sigma, 1 / c}\left(\frac{w}{c}\right)\right], \\
& \frac{1}{n} \sum_{j=1}^{n} \kappa_{4}\left(A_{N}\right)_{i j}\left[\operatorname{Re} B_{N}^{(m)}(z)\right]_{j j}\left[\operatorname{Im} B_{N}^{(m)}(w)\right]_{j j} \\
& \longrightarrow \kappa_{4}(i) \operatorname{Re}\left[\frac{z}{c} g_{\sigma, 1 / c}\left(\frac{z}{c}\right)\right] \operatorname{Im}\left[\frac{w}{c} g_{\sigma, 1 / c}\left(\frac{w}{c}\right)\right],
\end{aligned}
$$

in probability as $N \rightarrow \infty$. Indeed, for the first statement, by the triangle inequality

$$
\begin{aligned}
& \mid\left[\operatorname{Re} B_{N}^{(m)}(z)\right]_{j j} {\left[\operatorname{Re} B_{N}^{(m)}(w)\right]_{j j}-\operatorname{Re}\left[\frac{z}{c} g_{\sigma, 1 / c}\left(\frac{z}{c}\right)\right] \operatorname{Re}\left[\frac{w}{c} g_{\sigma, 1 / c}\left(\frac{w}{c}\right)\right] \mid } \\
& \leqslant \frac{|w|}{|\operatorname{Im} w|}\left|\left[\operatorname{Re} B_{N}^{(m)}(z)\right]_{j j}-\operatorname{Re}\left[\frac{z}{c} g_{\sigma, 1 / c}\left(\frac{z}{c}\right)\right]\right| \\
& \quad+\frac{|z|}{|\operatorname{Im} z|}\left|\left[\operatorname{Re} B_{N}^{(m)}(w)\right]_{j j}-\operatorname{Re}\left[\frac{w}{c} g_{\sigma, 1 / c}\left(\frac{w}{c}\right)\right]\right| .
\end{aligned}
$$

By Proposition 3.1, we obtain

$$
\left[\operatorname{Re} B_{N}^{(m)}(z)\right]_{j j}\left[\operatorname{Re} B_{N}^{(m)}(w)\right]_{j j}=\operatorname{Re}\left[\frac{z}{c} g_{\sigma, 1 / c}\left(\frac{z}{c}\right)\right] \operatorname{Re}\left[\frac{w}{c} g_{\sigma, 1 / c}\left(\frac{w}{c}\right)\right]+o(1) .
$$


The claim is then complete by assumption (5.1). The other two statements follow from the same argument.

Fix $p \geqslant 1$ and consider $z_{1}, \ldots, z_{p} \in \mathbb{C} \backslash \mathbf{R}$. We define the family of matrices

$$
C_{N}^{s, t}=\sum_{l=1}^{p}\left[a_{s, t}^{(l)} \operatorname{Re} B_{N}^{(m)}\left(z_{l}\right)+b_{s, t}^{(l)} \operatorname{Im} B_{N}^{(m)}\left(z_{l}\right)\right], \quad 1 \leqslant s, t \leqslant m
$$

where $a_{s, t}^{(l)}$ and $b_{s, t}^{(l)}$ are arbitrary real constants for $1 \leqslant s \leqslant t \leqslant m, 1 \leqslant l \leqslant p$. We now apply the central limit theorem for quadratic forms (see the appendix of [17]) to the family of matrices $C_{N}^{s, t}$ and use the above computations to conclude that $\Gamma_{N}(z)$ converges in finite dimensional distributions to $\sqrt{c} Y(z)$ for $\operatorname{Im} z \neq 0$.

For $z \in \mathbf{R} \backslash\left[0, \sigma^{2}(1+\sqrt{c})^{2}\right]$, define

$$
\delta=\operatorname{dist}\left(z,\left[0, \sigma^{2}(1+\sqrt{c})^{2}\right]\right) / 3 .
$$

Let $h(x)$ be a smooth function with compact support, where

$$
\begin{array}{ll}
h(x)=0 & \text { for } x \notin\left[-2 \delta, \sigma^{2}(1+\sqrt{c})^{2}+2 \delta\right], \\
h(x)=1 & \text { for } x \in\left[-\delta, \sigma^{2}(1+\sqrt{c})^{2}+\delta\right] .
\end{array}
$$

To complete the proof for $z \in \mathbf{R} \backslash\left[0, \sigma^{2}(1+\sqrt{c})^{2}\right]$, we repeat the same arguments as above replacing $B_{N}^{(m)}$ with $h\left(X_{N} X_{N}^{*}\right) B_{N}^{(m)}$, where $h$ is a $C^{\infty}(\mathbf{R})$ function with compact support such that

$$
h(x):=1 \quad \text { for } x \in\left[-\delta, \sigma^{2}(1+\sqrt{c})^{2}+\delta\right],
$$

for some $\delta>0$. It is essential here that

$$
\mathbf{P}\left(B_{N}^{(m)} \neq h\left(X_{N} X_{N}^{*}\right) B_{N}^{(m)}\right) \longrightarrow 0
$$

as $N \rightarrow \infty$ by Lemma 2.2. Theorem 5.1 is proved.

6. Fluctuations of matrix entires for regular functions. We now prove Theorem 1.2, Theorem 1.3 follows similarly.

$\mathrm{P}$ r o o f. In Theorem 5.1, Theorem 1.2 is proved for functions of the form

$$
\sum_{l=1}^{k} a_{l} \frac{1}{z_{l}-x}, \quad z_{l} \notin\left[0, \sigma^{2}(1+\sqrt{c})^{2}\right], \quad 1 \leqslant l \leqslant k .
$$

It follows from (1.8) and (5.4) that the limiting variance for functions of the form (6.1) given in Theorem 5.1 coincides with the one given in Theorem 1.2. We recall that

$$
\sqrt{N}\left(R_{N}(z)_{i j}-\delta_{i j} g_{\sigma, c_{N}}(z)\right), \quad 1 \leqslant i, j \leqslant m,
$$


converges in finite-dimensional distributions to the random point field $\sqrt{c} \times$ $g_{\sigma, c}^{2}(z) Y_{i j}(z), 1 \leqslant i, j \leqslant m$. For the off-diagonal entries $i \neq j$, one has

$$
\begin{aligned}
& g_{\sigma, c}^{2}(z) g_{\sigma, c}^{2}(w) \operatorname{Cov}\left(Y_{i j}(z), Y_{i j}(w)\right)=g_{\sigma, c}^{2}(z) g_{\sigma, c}^{2}(w) \sigma^{4} \varphi\left(\frac{z}{c}, \frac{w}{c}\right) \\
& =-\sigma^{4} g_{\sigma, c}^{2}(z) g_{\sigma, c}^{2}(w) \frac{z w\left(g_{\sigma, c}(z)-g_{\sigma, c}(w)\right)}{c(z-w)}-\sigma^{4} \frac{1-c}{c} g_{\sigma, c}^{2}(z) g_{\sigma, c}^{2}(w) \\
& =-\sigma^{4} g_{\sigma, c}(z) g_{\sigma, c}(w) \frac{1}{c}\left((c-1) g_{\sigma, c}(w) g_{\sigma, c}(z)+\frac{z g_{\sigma, c}(z)-w g_{\sigma, c}(w)}{\sigma^{2}(z-w)}\right) \\
& =-\sigma^{4} \frac{1}{c \sigma^{4}} g_{\sigma, c}(z) g_{\sigma, c}(w)-\sigma^{4} \frac{g_{\sigma, c}(z)-g_{\sigma, c}(w)}{c \sigma^{4}(z-w)} \\
& =\frac{1}{c} \operatorname{Cov}\left(\frac{1}{z-\eta}, \frac{1}{w-\eta}\right) .
\end{aligned}
$$

The calculations in the diagonal case $i=j$ are similar. To verify that the fourth cumulant term in $\operatorname{Cov}\left(Y_{i i}(z), Y_{i i}(w)\right)$ gives the required contribution (1.13), one uses the identity

$$
\begin{aligned}
\frac{z}{c} g_{\sigma, c}^{2}(z) g_{\sigma, 1 / c}\left(\frac{z}{c}\right) & =\frac{1}{c \sigma^{2}}\left(z g_{\sigma, c}^{2}(z)-g_{\sigma, c}\right) \\
& =\frac{1}{c \sigma^{2}}\left(\frac{\left(z-\sigma^{2}(c-1)\right) g_{\sigma, c}(z)-1}{\sigma^{2}}-g_{\sigma, c}\right) \\
& =\frac{1}{c \sigma^{2}} \frac{\left(z-c \sigma^{2}\right) g_{\sigma, c}(z)-1}{\sigma^{2}} .
\end{aligned}
$$

Thus, Theorem 1.2 is proved for functions of the form (6.1). By Lemma 2.2, the result then also follows for test functions of the form

$$
\sum_{l=1}^{k} a_{l} h_{l}(x) \frac{1}{z_{l}-x}, \quad z_{l} \notin\left[0, \sigma^{2}(1+\sqrt{c})^{2}\right], \quad 1 \leqslant l \leqslant k,
$$

where $h_{l}, 1 \leqslant l \leqslant k$, are $C^{\infty}(\mathbf{R})$ functions with compact support that satisfy (5.7).

By the Stone-Weierstrass theorem (see, e.g., [21]), functions (6.1) are dense in $C^{p}(X)$, for any compact set $X \subset \mathbf{R}$ and $p=0,1,2, \ldots$ Let $f$ be a $C^{\infty}(\mathbf{R})$ function such that $\operatorname{supp}(f) \subset[-L, L]$ for some $L>0$. Then there exists a sequence of functions $f_{j}$ of the form (6.2) that converge to $f$ in the $C^{4}[-L, L]$ norm (for the definition of the $C^{4}[-L, L]$ norm see (1.10)). Then, $f_{j}$ also converges to $f$ in the $\mathscr{H}_{s}$ norm for $s \leqslant 4$. By the estimate (4.6)

$$
\mathbb{V}\left[\sqrt{N}\left(f\left(X_{N}\right)_{i j}-f_{k}\left(X_{N}\right)_{i j}\right)\right] \leqslant \text { const }\left\|f(x)-f_{k}(x)\right\|_{s} .
$$

Since the right-hand side can be made arbitrarily small, and $\omega^{2}(f), \rho^{2}(f)$ in (1.12), (1.13) are continuous in the $\mathscr{H}_{s}$ norm, we obtain that $\sqrt{N}\left(f\left(X_{N}\right)_{i j}-\mathbf{E}\left[f\left(X_{N}\right)_{i j}\right]\right)$ converges in distribution to the Gaussian random variable defined in Theorem 1.2. Since smooth functions with compact 
support are dense in $\mathscr{H}_{s}$, the result can be extended to an arbitrary $f \in \mathscr{H}_{s}$. Theorem 1.2 is proved.

\section{REFERENCES}

1. Anderson G.W., Guionnet A., Zeitouni O. An Introduction to Random Matrices. Cambridge: Cambridge University Press, 2010, 492 p.

2. Bai Z. D. Methodologies in spectral analysis of large-dimensional random matrices, a review. - Statist. Sinica, 1999, v. 9, № 3, p. 611-677.

3. Bai Z. D., Silverstein J. Spectral Analysis of Large Dimensional Random Matrices, 2nd ed. New York: Springer, 2010, 551 p.

4. Bai Z. D., Silverstein J. W. No eigenvalues outside the support of the limiting spectral distribution of large-dimensional sample covariance matrices. - Ann. Probab., 1998, v. 26 , № 1 , p. $316-345$.

5. Ben Arous G., Guionnet A. Wigner matrices. In: Oxford Handbook on Random Matrix Theory, ed. by Akemann G., Baik J. and Di Francesco P., Oxford University Press, New York, 2011.

6. Billingsley P. Probability and Measure, 3rd ed. New York: Wiley, 1995, 593 p.

7. Davies E. B. The functional calculus. - J. London Math. Soc., 1995, v. 52, № 1, p. $166-176$.

8. D'Aristotile A., Diaconis P., Newman C. Brownian motion and the classical groups. - IMS Lecture Notes, Monograph Series, v. 41, p. 97-116, Inst. Math. Statist., Beachwood, 2003.

9. Durrett $R$. Probability: Theory and Examples, 4th ed. Cambridge: Cambridge University Press, 2010, 428 p.

10. Helffer B., Sjöstrand J. Equation de Schrödinger avec champ magnetique et equation de Harper, Schrödinger Operators. - Lecture Notes in Phys., 1989, v. 345, p. 118197.

11. Jiang T. How many entries of a typical orthogonal matrix can be approximated by independent normals? - Ann. Probab., 2006, v. 34, № 4, p. 1497-1529.

12. Jiang T. The entries of Haar-invariant matrices from the classical compact groups. J. Theor. Probab., 2010, v. 23, № 4, p. 1227-1243.

13. Khorunzhy A., Khoruzhenko B., Pastur L. Asymptotic properties of large random matrices with independent entries. - J. Math. Phys., 1996, v. 37, №10, p. 50335060.

14. Lytova A., Pastur L. Fluctuations of matrix elements of regular functions of Gaussian random matrices. - J. Stat. Phys., 2009, v. 134, № 1, p. 147-159.

15. Lytova A., Pastur L. Central limit theorem for linear eigenvalue statistics of random matrices with independent entries. - Ann. Probab., 2009, v. 37, № 5, p. 1778-1840.

16. Марченко В. А., Пастур Л. А. Распределение собственных значений в некоторых ансамблях случайных матриц. - Матем. сб., 1967, т. 72, № 114, с. 507-536.

17. O'Rourke S., Renfrew D., Soshnikov A. On fluctuations of matrix entries of regular functions of Wigner matrices with non-identically distributed entries. - J. Theor. Probab., 2013, v. 26, № 3, p. 750-780.

18. Pastur L., Lytova A. Non-Gaussian limiting laws for the entries of regular functions of the Wigner matrices, available at arXiv:1103.2345 [math.PR].

19. Pizzo A., Renfrew D., Soshnikov A. Fluctuations of matrix entries of regular functions of Wigner matrices. - J. Stat. Phys., 2012, v. 146, № 3, p. 550-591.

20. Pizzo A., Renfrew D., Soshnikov A. On finite rank deformations of Wigner matrices. - Ann. Inst. H. Poincaré Probab. Stat., 2013, v. 49, № 1, p. 64-94.

21. Reed M., Simon B. Methods of Modern Mathematical Physics, Vol. 1: Functional Analysis, 2nd ed. New York: Academic Press, 1980.

22. Shcherbina M.Central limit theorem for linear eigenvalue statistics of Wigner and sample covariance random matrices. - Журн. матем. физ., анал., геом., 2011, т. 7, № 2, c. 176-192. 
23. Shcherbina M. Letter from March 1, 2011.

24. Wigner E. On the distribution of the roots of certain symmetric matrices. - Ann. Math., 1958, v. 67, p. 325-327.

Поступила в редакцию

4.VI.2011

Исправленный вариант

1.II.2012 